\title{
Identification of the Dominant Genotypes of Phytophthora infestans in Canada Using Real-Time PCR with ASO-PCR Assays
}

\author{
Marie-Claude Gagnon, Canadian Food Inspection Agency (CFIA), Ottawa, ON, Canada; Lawrence Kawchuk, Agriculture and Agri-Food \\ Canada (AAFC), Lethbridge, AB, Canada; D. Mathieu Tremblay and Odile Carisse, AAFC, St-Jean-sur-Richelieu, QC, Canada; Giovanna \\ Danies and William E. Fry, Department of Plant Pathology and Plant-Microbe Biology, Cornell University, Ithaca, NY; C. André Lévesque,
} AAFC, Ottawa, ON, Canada; and Guillaume J. Bilodeau, CFIA, Ottawa

\begin{abstract}
Gagnon, M.-C., Kawchuk, L., Tremblay, D. M., Carisse, O., Danies, G., Fry, W. E., Lévesque, C. A., and Bilodeau, G. J. 2016. Identification of the dominant genotypes of Phytophthora infestans in Canada using real-time PCR with ASO-PCR assays. Plant Dis. 100:1482-1491.

Phytophthora infestans, a pathogenic oomycete that is the causal agent of potato and tomato late blight, has devastating effects worldwide. The genetic composition of $P$. infestans populations in Canada has changed considerably over the last few years, with the appearance of several new genotypes showing different mating types and sensitivity to the fungicide metalaxyl. Genetic markers allowing for a rapid assessment of genotypes from small amounts of biological material would be beneficial for the early detection and control of this pathogen throughout Canada. Mining of the $P$. infestans genome revealed several regions containing single-nucleotide polymorphisms (SNP) within both nuclear genes and flanking sequences of microsatellite loci. Allele-specific oligonucleotide

polymerase chain reaction (ASO-PCR) assays were developed from 14 of the 50 SNP found by sequencing. Nine optimized ASO-PCR assays were validated using a blind test comprising $P$. infestans and other Phytophthora spp. The assays revealed diagnostic profiles unique to each of the five dominant genotypes present in Canada. The markers developed in this study can be used with environmental samples such as infected leaves, and will contribute to the genomic toolbox available to assess the genetic diversity of $P$. infestans at the intraspecific level. For late blight management, early warning about $P$. infestans genotypes present in potato and tomato fields will help growers select the most appropriate fungicides and application strategies.
\end{abstract}

Phytophthora infestans (Mont.) de Bary is a pathogenic oomycete with devastating effects globally. It is the causal agent of potato (Solanum tuberosum) and tomato (S. lycopersicum) late blight, diseases for which outbreaks or epidemics have occurred in many countries since the 19th century (Bourke 1964; Fry 2008). This pathogen is notably responsible for the Irish potato famine in 1845 , and still has significant effects on potato and tomato production today (Bourke 1964; Fry 2008; Haverkort et al. 2008; Nowicki et al. 2012).

$P$. infestans is a heterothallic, diploid microorganism that can reproduce both clonally and sexually (Fry 2008). Clonal reproduction is commonly observed in North America, South America, Asia, Africa, and parts of Europe (Fry 2008; Fry et al. 2009). It was believed that sexual reproduction of this organism was mainly restricted to Scandinavia and the Toluca Valley in Mexico (Brurberg et al. 2011; Fry 2008; Fry et al. 2009; Grünwald and Flier 2005; Sjöholm et al. 2013; Yuen and Andersson 2013). However, recent evidence for recombination suggests that sexual reproduction might be contributing to the population structure of this pathogen in other parts of the world, including the United States and Canada (Danies et al. 2014; Kalischuk et al. 2012; Peters et al. 2014).

Many types of tools are available to characterize phenotypic and genetic diversity in P. infestans (Cooke and Lees 2004). Among the most widely used are mating type (A1 or A2), sensitivity to the fungicide metalaxyl (Dowley and O'Sullivan 1981), allozymes (Goodwin et al. 1994), restriction fragment length polymorphism (RFLP)

Corresponding author: G. J. Bilodeau;

E-mail: guillaume.bilodeau@inspection.gc.ca

GenBank accession numbers of DNA sequences: KM522922 to KM523128.

*The $\boldsymbol{e}$-Xtra logo stands for "electronic extra" and indicates that six supplementary tables are published online.

Accepted for publication 31 December 2015.

http://dx.doi.org/10.1094/PDIS-07-15-0763-RE

(c) 2016 The American Phytopathological Society fingerprints with the RG57 probe (Goodwin et al. 1992), mitochondrial haplotypes (Carter et al. 1990; Gavino and Fry 2002; Griffith and Shaw 1998), and microsatellites (Knapova and Gisi 2002; Lees et al. 2006; $\mathrm{Li}$ et al. 2010, 2013). These techniques are routinely employed to detect new clonal lineages or genotypes, to characterize $P$. infestans populations, to uncover recombination and sexual reproduction, and to document migration events (Cooke and Lees 2004; Fry 2008; Fry et al. 2009). Despite their usefulness, most of these tools share a common disadvantage that complicates the characterization of $P$. infestans diversity. With the exception of microsatellites, the methods all require the culturing of $P$. infestans on appropriate media before testing, which is time consuming and may sometimes be unsuccessful. Although microsatellites have good resolving power and can amplify from small amounts of DNA, technical setbacks such as protocol standardization and scoring calibration have reduced their portability and usage across laboratories (Guichoux et al. 2011).

Single-nucleotide polymorphisms (SNP) are biallelic, codominant genetic markers that result from point mutations producing single base-pair differences among chromosome sequences in a genome (Brumfield et al. 2003). These markers have several advantages such as their abundance in the genome, easiness to score, and potential amenability to different typing methods such as real-time polymerase chain reaction (PCR) assays (Brumfield et al. 2003; Guichoux et al. 2011; Morin et al. 2004). In recent years, real-time PCR assays have shown potential for sensitive and specific detection of Phytophthora spp. (Bilodeau et al. 2007b, 2014; Martin et al. 2009, 2012a). Allelespecific oligonucleotide (ASO)-PCR assays designed from SNP (Bottema and Sommer 1993; Green et al. 1991; Newton et al. 1989) and used with real-time PCR technology are very sensitive tests that can be used with samples containing small amounts of mixed DNA, such as environmental samples, making them well suited for rapid genotyping of plant pathogens (Bilodeau et al. 2007a; Gagnon et al. 2014).

Major genetic changes have been observed in populations of $P$. infestans in Canada over the last 30 years. Prior to the mid1990s, populations of this pathogen were mostly composed of a single genotype (US-1) that is sensitive to metalaxyl and has only the A1 mating type (Goodwin et al. 1994, 1996). During the 1990s, a new genotype (US-8) appeared in Canada. This genotype, with the A2 
Table 1. Isolates of Phytophthora infestans and other Phytophthora spp. used for sequencing (Seq), development (Dev), validation (Val), and standard curves (SC) or inoculation experiments with allele-specific oligonucleotide polymerase chain reaction (ASO-PCR) assays ${ }^{\text {a }}$

\begin{tabular}{|c|c|c|c|c|c|c|c|c|c|}
\hline Isolate & Species & Origin & Year & Source $^{b}$ & Genotype $^{c}$ & Seq & Dev & Val & $S^{d}$ \\
\hline LRC-1 & Phytophthora infestans & Canada & 2011 & AAFC-L & US-8 & $\mathrm{X}$ & $\ldots$ & $\mathrm{X}$ & $\ldots$ \\
\hline LRC-2 & P. infestans & Canada & 2011 & AAFC-L & US-8 & $\mathrm{X}$ & $\ldots$ & $\mathrm{X}$ & $\ldots$ \\
\hline LRC-3 & $P$. infestans & Canada & 2011 & AAFC-L & US-8 & $\mathrm{X}$ & $\ldots$ & $\mathrm{X}$ & $\ldots$ \\
\hline LRC-4 & P. infestans & Canada & 2011 & AAFC-L & US-11 & $X$ & $\cdots$ & $\cdots$ & $\ldots$ \\
\hline LRC-5 & P. infestans & Canada & 2011 & AAFC-L & US-11 & $\mathrm{X}$ & $\ldots$ & $\mathrm{X}$ & $\ldots$ \\
\hline LRC-6 & P. infestans & Canada & 2011 & AAFC-L & US-11 & $X$ & $\ldots$ & $X$ & $\ldots$ \\
\hline LRC-7 & P. infestans & Canada & 2011 & AAFC-L & US-23 & $\mathrm{X}$ & $\ldots$ & $\ldots$ & $\ldots$ \\
\hline LRC-8 & P. infestans & Canada & 2011 & AAFC-L & US-23 & $X$ & $\ldots$ & $X$ & $\ldots$ \\
\hline LRC-9 & $P$. infestans & Canada & 2011 & AAFC-L & US-23 & $X$ & $\ldots$ & $X$ & $\ldots$ \\
\hline DAOM 238142 & $P$. infestans & Canada & 2009 & CCFC & - & $X$ & $\ldots$ & $\ldots$ & $\ldots$ \\
\hline P1296 & P. infestans & Ireland & 1980 & WPC & US-1 & $\mathrm{X}$ & $\ldots$ & $\mathrm{X}$ & $\ldots$ \\
\hline P6749 & P. infestans & Mexico & - & WPC & - & $X$ & $\ldots$ & $\ldots$ & $\ldots$ \\
\hline P8372 & $P$. infestans & United States & - & WPC & US-6 & $X$ & $\ldots$ & $X$ & $\ldots$ \\
\hline P9175 & P. infestans & United States & 1995 & WPC & US-11 & $\mathrm{X}$ & $\ldots$ & $\ldots$ & $\ldots$ \\
\hline P10106 & $P$. infestans & United States & 1994 & WPC & US-7 & $X$ & $\cdots$ & $\mathrm{X}$ & $\ldots$ \\
\hline P10119 & P. infestans & Canada & 1996 & WPC & US-13 & $X$ & $\ldots$ & $\ldots$ & $\ldots$ \\
\hline Pi-01 & P. infestans & Canada & 2012 & AAFC-L & US-23 & $\mathrm{X}$ & $\mathrm{X}$ & $\mathrm{X}$ & \\
\hline $\mathrm{Pi}-02$ & $P$. infestans & Canada & 2012 & AAFC-L & US-23 & $X$ & $\mathrm{X}$ & $\ldots$ & $\ldots$ \\
\hline $\mathrm{Pi}-03$ & P. infestans & Canada & 2012 & AAFC-L & US-23 & $X$ & $\ldots$ & $X$ & $\mathrm{X}$ \\
\hline $\mathrm{Pi}-04$ & $P$. infestans & Canada & 2012 & AAFC-L & US-11 & $X$ & $\mathrm{X}$ & $X$ & $\ldots$ \\
\hline $\mathrm{Pi}-05$ & $P$. infestans & Canada & 2012 & AAFC-L & US-11 & $X$ & $\mathrm{X}$ & $X$ & $\ldots$ \\
\hline Pi-06 & $P$. infestans & Canada & 2012 & AAFC-L & US-11 & $X$ & $\ldots$ & $X$ & $X$ \\
\hline Pi-07 & P. infestans & Canada & 2012 & AAFC-L & US-8 & $X$ & $X$ & $X$ & $\ldots$ \\
\hline $\mathrm{Pi}-08$ & $P$. infestans & Canada & 2012 & AAFC-L & US-8 & $X$ & $\mathrm{X}$ & $X$ & $\ldots$ \\
\hline Pi-09 & P. infestans & Canada & 2012 & AAFC-L & US-8 & $X$ & $\ldots$ & $\mathrm{X}$ & $\mathrm{X}$ \\
\hline Pi-10 & P. infestans & Canada & 2012 & AAFC-L & US-22 & $X$ & $\mathrm{X}$ & $\ldots$ & $\ldots$ \\
\hline Pi-11 & $P$. infestans & Canada & 2012 & AAFC-L & US-22 & $\mathrm{X}$ & $\mathrm{X}$ & $\ldots$ & $\ldots$ \\
\hline $\mathrm{Pi}-12$ & $P$. infestans & Canada & 2012 & AAFC-L & US-22 & $X$ & $\ldots$ & $X$ & $\mathrm{X}$ \\
\hline Pi-13 & P. infestans & Canada & 2012 & AAFC-L & US-24 & $\mathrm{X}$ & $\mathrm{X}$ & $X$ & $\ldots$ \\
\hline $\mathrm{Pi}-14$ & $P$. infestans & Canada & 2012 & AAFC-L & US-24 & $\mathrm{X}$ & $\mathrm{X}$ & $X$ & $\ldots$ \\
\hline Pi-15 & $P$. infestans & Canada & 2012 & AAFC-L & US-24 & $\mathrm{X}$ & $\ldots$ & $\ldots$ & $\mathrm{X}$ \\
\hline PiUS23NB & P. infestans & Canada & - & AAFC-S & US-23 & $\ldots$ & $\ldots$ & $\mathrm{X}^{\mathrm{e}}$ & $\ldots$ \\
\hline Pi2011-072NB & $P$. infestans & Canada & 2011 & AAFC-S & US-24 & $\cdots$ & $\cdots$ & $\mathrm{X}^{\mathrm{e}}$ & $\cdots$ \\
\hline US110093 & P. infestans & United States & 2011 & Cornell & GDT-06 & $\ldots$ & $\ldots$ & $\mathrm{X}$ & $\ldots$ \\
\hline US110074 & P. infestans & United States & 2011 & Cornell & GDT-04 & $\ldots$ & $\ldots$ & $\mathrm{X}$ & $\ldots$ \\
\hline US110072 & $P$. infestans & United States & 2011 & Cornell & GDT-07 & $\ldots$ & $\ldots$ & $X$ & $\ldots$ \\
\hline US100023 & $P$. infestans & Canada & 2010 & Cornell & GDT-13 & $\ldots$ & $\ldots$ & $X$ & $\ldots$ \\
\hline US110061 & $P$. infestans & United States & 2011 & Cornell & GDT-05 & $\ldots$ & $\ldots$ & $X$ & $\ldots$ \\
\hline US100033 & $P$. infestans & Canada & 2010 & Cornell & GDT-16 & $\ldots$ & $\ldots$ & $\mathrm{X}$ & $\ldots$ \\
\hline US100034 & P. infestans & United States & 2010 & Cornell & GDT-20 & $\ldots$ & $\cdots$ & $\mathrm{X}$ & $\ldots$ \\
\hline US110086 & $P$. infestans & United States & 2011 & Cornell & GDT-03 & $\ldots$ & $\ldots$ & $X$ & $\ldots$ \\
\hline US110085 & $P$. infestans & United States & 2011 & Cornell & GDT-11 & $\ldots$ & $\ldots$ & $\mathrm{X}$ & $\ldots$ \\
\hline US100029 & $P$. infestans & United States & 2010 & Cornell & GDT-12 & $\ldots$ & $\ldots$ & $X$ & $\ldots$ \\
\hline US100032 & $P$. infestans & Canada & 2010 & Cornell & GDT-15 & $\ldots$ & $\ldots$ & $X$ & $\ldots$ \\
\hline US110084 & $P$. infestans & United States & 2011 & Cornell & GDT-01 & $\cdots$ & $\cdots$ & $\mathrm{X}$ & $\cdots$ \\
\hline US100019 & P. infestans & United States & 2010 & Cornell & GDT-19 & $\ldots$ & $\ldots$ & $\mathrm{X}$ & $\ldots$ \\
\hline US110064 & $P$. infestans & United States & 2011 & Cornell & GDT-02 & $\ldots$ & $\ldots$ & $X$ & $\ldots$ \\
\hline US110092 & P. infestans & United States & 2011 & Cornell & GDT-17 & $\ldots$ & $\ldots$ & $X$ & $\cdots$ \\
\hline US110071 & $P$. infestans & United States & 2011 & Cornell & GDT-08 & $\ldots$ & $\ldots$ & $X$ & $\ldots$ \\
\hline US110082 & P. infestans & United States & 2011 & Cornell & GDT-09 & $\ldots$ & $\ldots$ & $X$ & \\
\hline 1351798 & P. pseudosyringae & United States & 2005 & CDFA & N/A & $\cdots$ & $\ldots$ & $X$ & $\ldots$ \\
\hline P3009 & P. mirabilis & Mexico & 1987 & WPC & N/A & $\ldots$ & $\ldots$ & $\mathrm{X}$ & $\ldots$ \\
\hline BR 255 & P. nicotianae & - & 1977 & CCFC & N/A & $\ldots$ & $\ldots$ & $X$ & $\ldots$ \\
\hline BR 529 & P. megasperma & United Kingdom & 1949 & CCFC & N/A & $\cdots$ & $\ldots$ & $\mathrm{X}$ & $\cdots$ \\
\hline BR 675 & P. cactorum & Canada & 1989 & CCFC & N/A & $\cdots$ & $\cdots$ & $\mathrm{X}$ & $\cdots$ \\
\hline CBS 101553 & $P$. ramorum & Germany & 1995 & CBS & N/A & $\ldots$ & $\ldots$ & $\mathrm{X}$ & $\ldots$ \\
\hline DAOM 236393 & $P$. quercina & Italy & 1996 & CCFC & N/A & $\ldots$ & $\ldots$ & $\mathrm{X}$ & $\ldots$ \\
\hline BR 518 & P. citricola & Taiwan & 1926 & $\mathrm{CCFC}$ & N/A & $\ldots$ & $\ldots$ & $\mathrm{X}$ & $\ldots$ \\
\hline
\end{tabular}

\footnotetext{
a Symbol: - indicates missing data (not available).

b AAFC-L = Agriculture and Agri-Food Canada, Lethbridge, AB, Canada; CCFC = Canadian Collection of Fungal Cultures, Agriculture and Agri-Food Canada, Ottawa, ON, Canada; WPC = World Phytophthora Collection, University of California, Riverside; AAFC-S = Agriculture and Agri-Food Canada, St-Jean-surRichelieu, QC, Canada; Cornell = Cornell University, Ithaca, NY; CDFA = California Department of Food and Agriculture, Sacramento; and CBS = Centraalbureau voor Schimmelcultures, Utrecht, The Netherlands.

${ }^{\mathrm{c}}$ Genotypes based on mating type, allozymes, DNA fingerprints using the RG57 probe, mitochondrial haplotypes, microsatellites, or sequencing of nuclear genes (Danies et al. 2014; Goodwin et al. 1992, 1994; Li et al. 2013; Martin et al. 2012b). N/A = not applicable because not $P$. infestans isolates.

d Standard curve of nine optimized ASO-PCR assays.

e Used in the inoculation experiments to test the nine optimized ASO-PCR assays with environmental material.
} 
mating type, is highly aggressive on potato and resistant to the fungicide metalaxyl (Goodwin et al. 1996, 1998; Kato et al. 1997). This $P$. infestans genotype dominated in potato fields for nearly 20 years (Fry et al. 2009). Since 2009, increased incidence of late blight in Canada has coincided with the appearance of several new genotypes (US-11, US-22, US-23, and US-24) comprising both mating types and showing different levels of sensitivity to metalaxyl (Kalischuk et al. 2012; Kawchuk et al. 2011; Peters et al. 2014). For example, it was observed in Canada that the US-11 genotype was resistant to metalaxyl while the US-22, US-23, and US-24 genotypes were sensitive to the fungicide (Kalischuk et al. 2012). The increase in frequency of late blight outbreaks, the presence of strains possessing different characteristics, and the co-occurrence of A1 and A2 mating types in some regions poses a challenge for late blight management. A new genotyping tool that can detect the pathogen from very small amounts of DNA and facilitate rapid identification of the dominant genotypes of $P$. infestans in Canada would be very useful for late blight management decisions, such as the choice of fungicide to apply based on knowledge of the genotype fungicide resistance level, and frequency of fungicide applications based on virulence of the identified genotype.

The objectives of this study were to (i) develop ASO-PCR assays for identification of the five dominant genotypes of $P$. infestans present in Canada, (ii) validate the optimized ASO-PCR assays using a blind test comprising $P$. infestans and other Phytophthora spp., (iii) validate the optimized ASO-PCR assays using potentially recombinant $P$. infestans isolates from the United States and Canada, and (iv) test the optimized ASO-PCR assays using infected plant material.

\section{Materials and Methods}

To achieve these objectives, SNP found in nuclear genes and flanking microsatellite regions that were variable among the five dominant Canadian genotypes of $P$. infestans were used to design primers that were then tested for their utility in ASO-PCR assays.

Sampling. $P$. infestans isolates representing genotypes from Canada and other countries were obtained as listed in Table 1. The clonal lineages or genotypes reported are based on mating type, allozymes (Goodwin et al. 1994), DNA fingerprints using the RG57 probe (Goodwin et al. 1992), mitochondrial haplotypes (Martin et al. 2012b), microsatellite loci (Li et al. 2013), or sequencing of nuclear genes (Danies et al. 2014).

Culturing and DNA extraction were performed by different persons (Table 1) following different protocols. The 2011 and 2012 $P$. infestans isolates from Canada were grown and extracted as described by Kalischuk et al. (2012). The 2010 and 2011 potentially recombinant $P$. infestans isolates from the United States and Canada were grown and extracted as described by Danies et al. (2013). DNA from other $P$. infestans isolates was extracted from frozen mycelia following the Wizard Magnetic DNA Purification System for food (Promega Corp., Madison, WI), with volumes of solutions adapted for mycelial material, and the KingFisher ml instrument (Thermo Fisher Scientific Inc., Waltham, MA). DNA concentration was measured with a Qubit (Life Technologies Inc., Grand Island, NY).

DNA sequencing and ASO primer design. Several genes and flanking microsatellite regions were amplified and sequenced to look for SNP that distinguish one or several of the dominant Canadian $P$. infestans genotypes (US-8, US-11, US-22, US-23, and US-24). Selected loci were PCR amplified using the primers listed in Table 2 and Supplementary Table S1.

For nuclear genes, amplification reactions were carried out in $25-\mu 1$ volumes with the following final concentrations: $1 \times$ buffer, $0.2 \mathrm{mM}$ $\mathrm{dNTP}, 0.2 \mu \mathrm{M}$ each primer, $2 \mathrm{mM} \mathrm{MgCl}, 0.04 \mathrm{U}$ of Platinum Taq DNA polymerase (Life Technologies Inc.), and approximately $2 \mathrm{ng}$

Table 2. Nuclear genes and microsatellite flanking regions (MFR) used for sequencing and single-nucleotide polymorphism (SNP) mining in Phytophthora infestans

\begin{tabular}{|c|c|c|c|c|c|c|c|c|}
\hline Name $^{\mathbf{a}}$ & Primer name & Primer sequence & $\mathbf{T}_{\mathbf{a}}\left({ }^{\circ} \mathbf{C}\right)^{\mathbf{b}}$ & $\begin{array}{c}\text { Scored } \\
\text { bp }\end{array}$ & $\begin{array}{l}\text { Total } \\
\text { SNP } \\
\end{array}$ & $\begin{array}{c}\text { Variable } \\
\text { SNPc }\end{array}$ & Source & GenBank $^{\mathbf{d}}$ \\
\hline \multicolumn{9}{|l|}{ Nuclear genes } \\
\hline \multirow[t]{2}{*}{$\mathrm{ARP} 2 / 3$} & ARP23_For & TAYCCGCCCTACAAGACG & 55 & 864 & 3 & 1 & Blair et al. (2012) & KM522965-KM522995 \\
\hline & ARP23_Rev & CTTCTGGGTCTTGGACTGGT & $\cdots$ & $\ldots$ & $\cdots$ & $\cdots$ & $\ldots$ & $\ldots$ \\
\hline \multirow[t]{2}{*}{ PUA } & PUA_For & AGGTCAAGTCCTCGCAGCAG & 67 & 583 & 7 & 7 & Blair et al. (2012) & KM522930-KM522960 \\
\hline & PUA_Rev & AGGTCGTCRCCMAGGAAGTG & $\ldots$ & $\ldots$ & $\ldots$ & $\ldots$ & & \\
\hline \multirow[t]{2}{*}{ Ras } & Ras_For & CGTGTCTGCTTCTCCGTTTCG & 55 & 549 & 6 & 6 & $\begin{array}{l}\text { Gómez-Alpizar } \\
\text { et al. (2007) }\end{array}$ & KM523098-KM523128 \\
\hline & Ras_Rev & CCAGGCTTTCGGCAAATTCC & & & ... & $\ldots$ & & \\
\hline \multirow[t]{2}{*}{ Ras intron 1} & RasInt_For & TTGCAGCACAACCCAAGACG & 55 & 288 & 6 & 6 & $\begin{array}{l}\text { Gómez-Alpizar } \\
\text { et al. (2007) }\end{array}$ & KM522996-KM523008 \\
\hline & RasInt_Rev & TGCACGTACTATTCGGGGTTC & $\ldots$ & $\ldots$ & $\ldots$ & $\ldots$ & $\ldots$ & \\
\hline \multirow[t]{2}{*}{ TRP1 } & Trp_For & GCCGCCAAGCAGGTCRT & 62 & 525 & 3 & 3 & Blair et al. (2012) & KM523017-KM523047 \\
\hline & Trp_Rev & RAYGCTGTTCACCTCSACCA & $\ldots$ & $\ldots$ & $\ldots$ & $\ldots$ & $\ldots$ & $\ldots$ \\
\hline \multicolumn{9}{|c|}{ ( } \\
\hline \multirow[t]{2}{*}{$3027 \mathrm{R}$} & 3027R_fseq & CCCACCGATCACTCCACCT & TD & 495 & 1 & 1 & Abbott et al. (2010) & KM522926-KM522929 \\
\hline & 3027R_rev & CGTGGGCAACTACATGACCG & $\ldots$ & $\ldots$ & $\ldots$ & $\cdots$ & $\ldots$ & $\ldots$ \\
\hline \multirow[t]{2}{*}{$3157 \mathrm{~F}$} & 3157F_for & CGCGATGATGTGGAAGATGG & $\mathrm{TD}$ & 507 & 3 & 3 & Abbott et al. (2010) & KM523013-KM523016 \\
\hline & 157F_rseq3 & TGTAAACCATGCTCTACGATT & $\ldots$ & $\ldots$ & $\cdots$ & $\ldots$ & $\ldots$ & $\ldots$ \\
\hline \multirow[t]{2}{*}{$3279 \mathrm{~F}$} & 3279F_fseq & TGTGTATATTTATCGAAAGCA & $\mathrm{TD}$ & 436 & 5 & 4 & Abbott et al. (2010) & KM523048-KM523066 \\
\hline & 3279F_rseq & GCAAAATCGCATTATGTGGT & $\ldots$ & $\ldots$ & $\ldots$ & $\ldots$ & $\ldots$ & $\ldots$ \\
\hline \multirow[t]{2}{*}{$3332 \mathrm{~F}$} & 3332F_for & ACTGAGACCACCACGCCACC & TD & 398 & 8 & 7 & Abbott et al. (2010) & KM523067-KM523097 \\
\hline & 3332F_rseq2 & АTCСТTCTCСТCСТTGTCCA & $\ldots$ & $\ldots$ & $\ldots$ & $\ldots$ & $\ldots$ & $\ldots$ \\
\hline \multirow[t]{2}{*}{$3345 \mathrm{~F}$} & 3345F_for & TGGAGATCGGCGTACCAAGG & $\mathrm{TD}$ & 360 & 5 & 5 & Abbott et al. (2010) & KM522961-KM522964 \\
\hline & 3345F_rseq & GGATGGGATGCTGTGCTTAG & $\ldots$ & $\ldots$ & $\ldots$ & $\ldots$ & $\ldots$ & $\ldots$ \\
\hline \multirow[t]{2}{*}{ 3350_9F } & 3350_9F_for & TGCGTTATTGTGCCACCCAC & TD & 477 & 3 & 3 & Abbott et al. (2010) & KM522922-KM522925 \\
\hline & 3350_9F_rseq2 & GGTCTATTTGCGCTTCTTCT & $\ldots$ & $\ldots$ & $\ldots$ & $\ldots$ & $\ldots$ & $\ldots$ \\
\hline \multirow[t]{2}{*}{ 3361_7R } & 3361_7R_fseq & GGCCTTTTTAAACGTTTGGA & TD & 420 & 5 & 5 & Abbott et al. (2010) & KM523009-KM523012 \\
\hline & 3361_7R_rseq & GCATTTCATTCAAACACAG & $\ldots$ & $\ldots$ & $\ldots$ & $\ldots$ & $\ldots$ & $\ldots$ \\
\hline Total & $\ldots$ & $\ldots$ & $\ldots$ & $\ldots$ & 55 & 51 & $\ldots$ & $\ldots$ \\
\hline
\end{tabular}

a ARP2/3 = actin-related protein 2/3 complex, PUA = conserved hypothetical protein, Ras = Rab 1 family GTPase PiYPT1, and TRP1 = N-(5'-phosphoribosyl) anthranilate isomerase indole-3-glycerol-phosphate synthase.

${ }^{\mathrm{b}}$ Annealing temperature. $\mathrm{TD}=$ touchdown: annealing temperature from 65 to $53^{\circ} \mathrm{C}$ (dropping $3^{\circ} \mathrm{C}$ per three cycles; see Material and Methods).

c SNP showing variation among the five dominant Canadian genotypes.

d GenBank accession numbers. 
of template DNA. PCR conditions were as follows: 2 min at $95^{\circ} \mathrm{C}$; 35 cycles of $30 \mathrm{~s}$ at $95^{\circ} \mathrm{C}, 30 \mathrm{~s}$ at the locus-specific annealing temperature (Table 2), and $1 \mathrm{~min}$ at $72^{\circ} \mathrm{C}$; and $5 \mathrm{~min}$ at $72^{\circ} \mathrm{C}$.

For flanking microsatellite regions, amplification reactions were carried out in 10- $\mu$ l volumes with the following final concentrations: $1 \times$ buffer, $0.1 \mathrm{mM}$ dNTP, $0.1 \mu \mathrm{M}$ each primer, $2 \mathrm{mM} \mathrm{MgCl}_{2}, 0.05 \mathrm{U}$ of Platinum Taq DNA polymerase (Life Technologies Inc.), and approximately $2 \mathrm{ng}$ of template DNA (Abbott et al. 2010). PCR conditions were $3 \mathrm{~min}$ at $95^{\circ} \mathrm{C}$; a "touchdown" of $30 \mathrm{~s}$ at $95^{\circ} \mathrm{C}$, $30 \mathrm{~s}$ at 65 to $53^{\circ} \mathrm{C}$ (dropping $3^{\circ} \mathrm{C}$ per three cycles), and $90 \mathrm{~s}$ at $72^{\circ} \mathrm{C} ; 25$ cycles of $30 \mathrm{~s}$ at $95^{\circ} \mathrm{C}, 30 \mathrm{~s}$ at $50^{\circ} \mathrm{C}$, and $90 \mathrm{~s}$ at $72^{\circ} \mathrm{C}$; and one final step of $3 \mathrm{~min}$ at $72^{\circ} \mathrm{C}$.

Loci that generated a single amplification product of the expected size (Table 2) were sequenced in both directions using $1 \mu l$ of the PCR product, BigDye Terminator v3.1 Cycle Sequencing Kit (Life Technologies Inc.) and $0.16 \mu \mathrm{M}$ primer in a $20-\mu 1$ reaction volume. Sequencing products were run on an ABI $3130 x l$ Genetic Analyzer (Life Technologies Inc.). Electropherograms were inspected visually to ensure proper base calling. Nucleotide positions that showed double peaks were scored as heterozygous. Sequences were aligned using BioEdit, version 7.2.0 (Hall 1999). Loci that failed to produce a single PCR product, contained indels, contained intralineage mutations, or were not variable were not investigated further.

For all successfully sequenced loci, a DNA sequence alignment that included samples of all dominant Canadian genotypes and samples of other P. infestans genotypes (Table 1) was used for SNP mining. SNP were considered candidates for ASO primer design if they distinguished one or more of the dominant Canadian genotypes. For each candidate SNP, a common primer and two ASO primers were designed. For a given SNP, ASO primers are identical except for the last base at the $3^{\prime}$ position. Primer selection criteria included a melting temperature of $66^{\circ} \mathrm{C}$, primer lengths of 18 to $22 \mathrm{bp}$, avoidance of secondary structures, and PCR products of less than $200 \mathrm{bp}$.

Optimization of ASO primer pairs. The real-time PCR conditions of the ASO primer pairs were optimized using two isolates of each dominant $P$. infestans Canadian genotype (US-8, US-11, US-22, US-23, and US-24) and a negative control template (Table 1). For each SNP tested, two separate PCR reactions were

Table 3. Primers and standard curve statistics for nine optimized allele-specific oligonucleotide polymerase chain reaction (ASO-PCR) assays developed for Phytophthora infestans

\begin{tabular}{|c|c|c|c|c|c|c|}
\hline Name & Primer sequence $^{\mathbf{a}}$ & $\mathbf{T}_{\mathbf{a}}\left({ }^{\circ} \mathbf{C}\right)^{\mathbf{b}}$ & $y=a x+y_{0}^{c}$ & $R^{2}$ & Efficiency & $\operatorname{LOD}(f g / \mu l)^{\mathrm{d}}$ \\
\hline ASO Arp23-182 A & GACGCTGTATTTGCTGAGATTA & 68 & $y=-3.42 x+18.71$ & 0.994 & 0.96 & 500 \\
\hline ASO Arp23-182 $\mathrm{T}^{\mathrm{e}}$ & GACGCTGTATTTGCTGAGATTT̄ & $\ldots$ & $y=-3.05 x+19.76$ & 0.987 & 1.13 & $\ldots$ \\
\hline RevCommonArp23-207f & CCCAAACACCAGAATACACGĀ̄TTAGC & $\ldots$ & $\ldots$ & $\ldots$ & $\ldots$ & $\ldots$ \\
\hline ASO PUA-120 Te & GTTGCCAAATGGTTAGTTATCT & 66 & $y=-3.72 x+21.63$ & 0.986 & 0.86 & 50 \\
\hline ASO PUA-120 $\mathrm{A}^{\mathrm{e}}$ & GTTGCCAAATGGTTAGTTATCA $\bar{A}$ & $\ldots$ & $y=-3.47 x+23.06$ & 0.983 & 0.94 & $\ldots$ \\
\hline RevCommonPUA-190 f & GTGCAACACGATCTGGATGTGG & $\ldots$ & $\ldots$ & $\ldots$ & $\ldots$ & $\ldots$ \\
\hline ASO PUA-225 Ce & CACGAAGGCGAGCCTC & 64 & $y=-3.25 x+18.32$ & 0.994 & 1.03 & 50 \\
\hline ASO PUA-225 Te & CACGAAGGCGAGCCTT̄ & $\ldots$ & $y=-3.28 x+21.20$ & 0.997 & 1.02 & $\ldots$ \\
\hline RevCommonPUA-260 f & GCAGCAGCTTGAGCGT̄GG & $\ldots$ & $\ldots$ & $\ldots$ & $\ldots$ & $\ldots$ \\
\hline ASO PUA-570 Ge & CATGTACGTGGTGTAATTATTTG & 64 & $y=-2.99 x+19.11$ & 0.987 & 1.16 & 50 \\
\hline ASO PUA-570 A & CATGTACGTGGTGTAATTATTTA & $\ldots$ & $y=-3.29 x+21.03$ & 0.982 & 1.01 & $\ldots$ \\
\hline RevCommonPUA-601 ${ }^{\mathrm{f}}$ & TCTTGTTGCGACTGACAGAAACATT & $\ldots$ & $\ldots$ & $\ldots$ & $\ldots$ & $\ldots$ \\
\hline Rev-ASO Ras-98 C $(\mathrm{G})^{\mathrm{f}}$ & CAATGTAATGGCAATCTAGACC & 64 & $y=-3.28 x+18.78$ & 0.992 & 1.02 & 50 \\
\hline Rev-ASO Ras-98 A(T) ${ }^{\mathrm{f}}$ & CAATGTAATGGCAATCTAGACĀ & $\ldots$ & $y=-3.29 x+19.64$ & 0.982 & 1.01 & $\ldots$ \\
\hline CommonRas- $41^{\mathrm{e}}$ & CATCGGTGTTGACTTTGTGAGTGT & $\ldots$ & $\ldots$ & $\ldots$ & $\ldots$ & $\ldots$ \\
\hline ASO Ras-376 Te & GGACCAGGAGTCGTTCAAT & 66 & $y=-3.14 x+17.10$ & 0.998 & 1.08 & 50 \\
\hline ASO Ras-376 Ce & GGACCAGGAGTCGTTCAA $\overline{\mathrm{C}}$ & $\ldots$ & $y=-3.33 x+17.47$ & 0.990 & 1.00 & $\ldots$ \\
\hline RevCommonRas- $468^{\mathrm{f}}$ & TCACAGGCGTATCTGCAAATCAAAGC & $\ldots$ & $\ldots$ & $\ldots$ & $\ldots$ & $\ldots$ \\
\hline RevASO 3332F-225 C(G) $)^{\mathrm{f}}$ & CCCTCGTGGGCTTCAC & 64 & $y=-3.33 x+20.15$ & 0.982 & 1.00 & 500 \\
\hline RevASO 3332F-225 G(C) ${ }^{\mathrm{f}}$ & CCCTCGTGGGCTTCA $\overline{\mathrm{G}}$ & $\ldots$ & $y=-3.64 x+22.29$ & 0.996 & 0.88 & $\ldots$ \\
\hline Common3332F-225e & CAAAGCGTGCACAGGTAACGTG & $\ldots$ & $\ldots$ & $\ldots$ & $\ldots$ & $\ldots$ \\
\hline RevASO 3332F-258 G(C), & TGGACCGGATCTTCGG & 64 & $y=-3.41 x+18.01$ & 0.991 & 0.96 & 500 \\
\hline RevASO 3332F-258 T(A) $)^{\mathrm{f}, \mathrm{g}}$ & TGGACCGGATCTTCGT & $\ldots$ & $y=-3.61 x+19.67$ & 0.990 & 0.89 & $\ldots$ \\
\hline RevASO 3332F-344 G(C) ${ }^{\mathrm{f}}$ & CTAAGCAATGCACGCTTG & 60 & $y=-3.36 x+17.24$ & 0.996 & 0.98 & 500 \\
\hline RevASO $3332 \mathrm{~F}-344 \mathrm{~A}(\mathrm{~T})^{\mathrm{f}}$ & CTAAGCAATGCACGCTTA & $\ldots$ & $y=-3.49 x+20.34$ & 0.997 & 0.93 & $\ldots$ \\
\hline Common3332F-344e & CGGTCCACGAAGACGGĀ & $\ldots$ & $\ldots$ & $\ldots$ & $\ldots$ & $\ldots$ \\
\hline
\end{tabular}

a Single-nucleotide polymorphisms (SNP) are underlined.

b Annealing temperature.

c Standard curve linear equation ( $\mathrm{a}=$ slope, $y_{0}=$ intercept at origin) to calculate the efficiency of the PCR.

${ }^{\mathrm{d}} \mathrm{LOD}=$ limit of detection.

e Forward primer.

${ }^{\mathrm{f}}$ Reverse primer.

g Primers used with the common primer Common3332F-225.

Table 4. Expected genotypes of the five dominant Canadian genotypes of Phytophthora infestans at nine optimized allele-specific oligonucleotide polymerase chain reaction (ASO-PCR) assays

\begin{tabular}{|c|c|c|c|c|c|c|c|c|c|}
\hline Genotype & $\begin{array}{c}\text { ASO } \\
\text { Arp23-182 }\end{array}$ & $\begin{array}{c}\text { ASO } \\
\text { PUA-120 }\end{array}$ & $\begin{array}{c}\text { ASO } \\
\text { PUA-225 }\end{array}$ & $\begin{array}{c}\text { ASO } \\
\text { PUA-570 }\end{array}$ & $\begin{array}{c}\text { RevASO } \\
\text { Ras-98 }\end{array}$ & $\begin{array}{c}\text { ASO } \\
\text { Ras-376 }\end{array}$ & $\begin{array}{c}\text { RevASO } \\
\text { 3332F-225 }\end{array}$ & $\begin{array}{c}\text { RevASO } \\
\text { 3332F-258 }\end{array}$ & $\begin{array}{c}\text { RevASO } \\
\text { 3332F-344 } \\
\end{array}$ \\
\hline US-8 & AA & TT & $\mathrm{CC}$ & AA & GG & $\mathrm{TC}$ & GG & $\mathrm{CC}$ & $\mathrm{CT}^{\mathrm{b}}$ \\
\hline US-11 & AA & TT & $\mathrm{CC}$ & GG & $\mathrm{GT}^{\mathrm{b}}$ & $\mathrm{TC}$ & GG & $\mathrm{CA}^{\mathrm{b}}$ & $\mathrm{CC}$ \\
\hline US-22 & $\mathrm{AT}^{\mathrm{b}}$ & $\mathrm{TT}$ & $\mathrm{CC}$ & GA & $\mathrm{GT}^{\mathrm{b}}$ & $\mathrm{TT}^{\mathrm{b}}$ & GG & $\mathrm{CC}$ & $\mathrm{CC}$ \\
\hline US-23 & $\mathrm{AT}^{\mathrm{b}}$ & $\mathrm{TA}^{\mathrm{b}}$ & $\mathrm{CT}^{\mathrm{b}}$ & GA & GG & $\mathrm{TC}$ & $\mathrm{GC}^{\mathrm{b}}$ & $\mathrm{CC}$ & $\mathrm{CC}$ \\
\hline US-24 & $\mathrm{AA}$ & $\mathrm{TT}$ & $\mathrm{CC}$ & AA & GG & $\mathrm{TC}$ & $\mathrm{GC}^{\mathrm{b}}$ & $\mathrm{CC}$ & $\mathrm{CC}$ \\
\hline
\end{tabular}

a The three genotypes at this ASO-PCR assay are diagnostic of $P$. infestans genotypes.

$\mathrm{b}$ Indicates the diagnostic genotypes at each of the ASO-PCR assays. 
conducted, one for each allele targeted. All reactions were carried out in $25-\mu$ l volumes with the following final concentrations: $1 \times$ of GoTaq qPCR Master Mix (Promega Corp.), $0.3 \mu \mathrm{M}$ each primer, and approximately $1 \mathrm{ng}$ of template DNA. PCR conditions were as follows: 3 min at $95^{\circ} \mathrm{C} ; 45$ cycles of $15 \mathrm{~s}$ at $94^{\circ} \mathrm{C}, 30 \mathrm{~s}$ at an annealing temperature between 60 and $68^{\circ} \mathrm{C}\left(2^{\circ} \mathrm{C}\right.$ increment per test), and $30 \mathrm{~s}$ at $72^{\circ} \mathrm{C}$; and $15 \mathrm{~s}$ at $95^{\circ} \mathrm{C}$ followed by a melting curve from 55 to $95^{\circ} \mathrm{C}$, with a reading every $1.0^{\circ} \mathrm{C}$ and a hold for a 6-s measurement. Fluorescence was also measured during the extension phase at $72^{\circ} \mathrm{C}$. All reactions were run on a Rotor-Gene 6000 Real-Time PCR machine (Corbett-Research, Montreal Biotech Inc., Dorval, QC, Canada) and data were analyzed with Rotor-Gene Q series software (version 1.7; Corbett-Research).

Validation of ASO primer pairs. To distinguish and identify all five dominant Canadian genotypes of $P$. infestans, a combination of nine ASO-PCR assays was validated using a blind test (Table 3) performed at the Canadian Food Inspection Agency (Ottawa, ON, Canada). The blind test consisted of 31 Phytophthora samples and included one negative control template, two to six isolates of each of the dominant Canadian P. infestans genotypes (US-8, US-11, US-22, US-23, and US-24), three other $P$. infestans genotypes (US-1, US-6, and US-7), as well as eight other Phytophthora spp. (Table 1). DNA concentrations ranged from $\leq 0.03$ to $1.0 \mathrm{ng} / \mu \mathrm{l}$. Samples were tested with the nine optimized ASO primer pairs, and an overall profile was established for each sample based on the genotypes and profiles obtained at each locus. For each of the loci tested, PCR assays were performed at the optimal annealing temperatures listed in Table 3, and data analyses were as described above.

Recombinant isolates from Canada and the eastern United States also were used to validate further the nine optimized ASO-PCR assays. In all, 17 recombinant $P$. infestans isolates and one negative control template were included in the test (Table 1). DNA concentrations ranged from 0.8 to $1.2 \mathrm{ng} / \mu \mathrm{l}$. Samples were tested with the nine optimized ASO primer pairs, and an overall profile was established for each sample based on the genotypes and profiles obtained at each locus. For each of the loci tested, PCR assays were performed at the optimal annealing temperatures listed in Table 3. Reactions were run on either a Rotor-Gene 6000 real-time PCR machine or a StepOne Real-Time PCR System. Data were analyzed with either Rotor-Gene Q series software, version 1.7 or StepOne Software, version 2.3.

Standard curves of ASO primer pairs. Serial dilutions of $P$. infestans DNA were used to produce standard curves for each targeted allele of each of the nine optimized ASO primer pairs to determine the limit of detection and sensitivity of the assays (Table 3). DNA serial dilutions consisted of one $P$. infestans isolate heterozygous for a specific primer pair (different isolates were used for the different primer pairs; Table 1) diluted in a series of 1:10 from

Table 5. Cycle threshold (Ct) values, genotypes, and overall profile for a blind panel of Phytophthora infestans and other Phytophthora spp. at each of nine allelespecific oligonucleotide polymerase chain reaction (ASO-PCR) assays ${ }^{\mathrm{a}}$

\begin{tabular}{|c|c|c|c|c|c|c|c|c|c|c|c|c|c|c|c|}
\hline \multirow[b]{2}{*}{ Sample } & \multirow[b]{2}{*}{ Isolate } & \multirow[b]{2}{*}{ Species } & \multirow[b]{2}{*}{ Genotype } & \multicolumn{3}{|c|}{ ASO Arp23-182 } & \multicolumn{3}{|c|}{ ASO PUA-120 } & \multicolumn{3}{|c|}{ ASO PUA-225 } & \multicolumn{3}{|c|}{ ASO PUA-570 } \\
\hline & & & & $\mathbf{A}$ & $\mathbf{T}$ & Type $^{\text {b }}$ & $\mathbf{T}$ & $\mathbf{A}$ & Type & $\mathbf{C}$ & $\mathbf{T}$ & Type & G & $\mathbf{A}$ & Type \\
\hline 1 & $\mathrm{Pi}-08$ & Phytophthora infestans & US-8 & 20.9 & $32.4^{\mathrm{d}}$ & AA & 21.6 & - & TT & 21.5 & 30.7 & $\mathrm{CC}$ & 37.6 & 22.6 & AA \\
\hline 2 & $\mathrm{Pi}-04$ & P. infestans & US-11 & 21.7 & - & $\mathrm{AA}$ & 21.6 & $38.6^{\mathrm{d}}$ & $\mathrm{TT}$ & 22.1 & $31.9^{\mathrm{d}}$ & $\mathrm{CC}$ & 22.4 & 34.9 & GG \\
\hline 3 & Pi-03 & P. infestans & US-23 & 22.7 & 23.5 & $\mathrm{AT}^{\mathrm{e}}$ & 22.6 & 23.7 & $\mathrm{TA}^{\mathrm{e}}$ & 22.9 & 24.9 & $\mathrm{CT}^{\mathrm{e}}$ & 24.5 & 24.0 & GA \\
\hline 4 & LRC-9 & P. infestans & US-23 & 23.8 & 24.4 & $\mathrm{AT}^{\mathrm{e}}$ & 23.3 & 24.3 & $\mathrm{TA}^{\mathrm{e}}$ & 23.5 & 25.4 & $\mathrm{CT}^{\mathrm{e}}$ & 25.0 & 25.0 & GA \\
\hline 5 & LRC-2 & P. infestans & US-8 & 25.5 & $40.0^{\mathrm{d}}$ & $\mathrm{AA}$ & 24.8 & $36.2^{\mathrm{d}}$ & $\mathrm{TT}$ & 25.4 & $35.4^{\mathrm{d}}$ & $\mathrm{CC}$ & $33.6^{\mathrm{d}}$ & 26.6 & AA \\
\hline 6 & LRC-3 & P. infestans & US-8 & 24.8 & - & AA & 24.5 & - & TT & 24.9 & 33.7 & $\mathrm{CC}$ & 39.8 & 26.2 & AA \\
\hline 7 & P10106 & P. infestans & US-7 & 27.3 & - & AA & 26.6 & - & TT & 27.2 & $36.2^{\mathrm{d}}$ & $\mathrm{CC}$ & - & 28.5 & AA \\
\hline 8 & $\mathrm{Pi}-13$ & P. infestans & US-24 & 22.6 & - & $\mathrm{AA}$ & 22.1 & $40.3^{\mathrm{d}}$ & $\mathrm{TT}$ & 22.6 & $31.8^{\mathrm{d}}$ & $\mathrm{CC}$ & 32.8 & 24.0 & AA \\
\hline 9 & $\mathrm{Pi}-12$ & P. infestans & US-22 & 21.5 & 21.3 & $\mathrm{AT}^{\mathrm{e}}$ & 20.2 & $35.5^{\mathrm{d}}$ & TT & 20.5 & $30.6^{\mathrm{d}}$ & $\mathrm{CC}$ & 22.0 & 23.0 & GA \\
\hline 10 & Pi-14 & P. infestans & US-24 & 22.8 & $34.4^{\mathrm{d}}$ & AA & 22.2 & $38.9^{\mathrm{d}}$ & TT & 22.9 & $33.4^{\mathrm{d}}$ & $\mathrm{CC}$ & 30.6 & 24.3 & $\mathbf{A A}$ \\
\hline 11 & $\mathrm{Pi}-05$ & P. infestans & US-11 & 21.8 & - & AA & 21.8 & $32.2^{\mathrm{d}}$ & $\mathrm{TT}$ & 22.0 & $31.5^{\mathrm{d}}$ & $\mathrm{CC}$ & 22.4 & $44.4^{\mathrm{d}}$ & GG \\
\hline 12 & 1351798 & P. pseudosyringae & N/A & - & - & N/A & - & $39.4^{\mathrm{d}}$ & N/A & $30.7^{\mathrm{d}}$ & $34.2^{\mathrm{d}}$ & N/A & - & - & N/A \\
\hline 13 & BR 675 & P. cactorum & N/A & - & $38.3^{\mathrm{d}}$ & N/A & - & - & N/A & $27.0^{\mathrm{d}}$ & $27.2^{\mathrm{d}}$ & N/A & - & - & N/A \\
\hline 14 & LRC-5 & P. infestans & US-11 & 27.3 & $32.3^{\mathrm{d}}$ & $\mathrm{AA}$ & 26.0 & - & $\mathrm{TT}$ & 26.4 & $38.5^{\mathrm{d}}$ & $\mathrm{CC}$ & 25.9 & - & GG \\
\hline 15 & P1296 & P. infestans & US-1 & 25.3 & $35.3^{\mathrm{d}}$ & AA & 27.6 & 27.0 & $\mathrm{TA}^{\mathrm{e}}$ & 27.5 & 27.0 & $\mathrm{CT}^{\mathrm{e}}$ & 26.7 & - & GG \\
\hline 16 & $\mathrm{Pi}-06$ & P. infestans & US-11 & 21.9 & - & AA & 21.7 & $36.7^{\mathrm{d}}$ & $\mathrm{TT}$ & 21.9 & 31.0 & $\mathrm{CC}$ & 22.4 & $42.8^{\mathrm{d}}$ & GG \\
\hline 17 & Pi-10 & P. infestans & US-22 & 21.2 & 21.0 & $\mathrm{AT}^{\mathrm{e}}$ & 20.1 & $35.2^{\mathrm{d}}$ & $\mathrm{TT}$ & 20.3 & $30.5^{\mathrm{d}}$ & $\mathrm{CC}$ & 21.7 & 22.7 & GA \\
\hline 18 & $\mathrm{Pi}-09$ & P. infestans & US-8 & 21.1 & $40.0^{\mathrm{d}}$ & AA & 21.2 & $39.1^{\mathrm{d}}$ & TT & 21.6 & $31.3^{\mathrm{d}}$ & $\mathrm{CC}$ & 34.4 & 23.2 & AA \\
\hline 19 & P8372 & P. infestans & US-6 & 25.7 & - & AA & 25.3 & - & TT & 25.2 & $38.4^{\mathrm{d}}$ & $\mathrm{CC}$ & 26.2 & - & GG \\
\hline 20 & BR 255 & P. nicotianae & N/A & - & $33.3^{\mathrm{d}}$ & N/A & $32.7^{\mathrm{d}}$ & - & N/A & $31.0^{\mathrm{d}}$ & $34.2^{\mathrm{d}}$ & N/A & - & - & N/A \\
\hline 21 & BR 529 & P. megasperma & N/A & - & $32.4^{\mathrm{d}}$ & N/A & - & - & N/A & $33.4^{\mathrm{d}}$ & $29.2^{\mathrm{d}}$ & N/A & - & - & N/A \\
\hline 22 & LRC-8 & P. infestans & US-23 & 25.6 & 25.9 & $\mathrm{AT}^{\mathrm{e}}$ & 24.5 & 25.2 & $\mathrm{TA}^{\mathrm{e}}$ & 25.6 & 27.6 & $\mathrm{CT}^{\mathrm{e}}$ & 26.1 & 26.1 & GA \\
\hline 23 & $\mathrm{Pi}-01$ & P. infestans & US-23 & 22.7 & 23.8 & $\mathrm{AT}^{\mathrm{e}}$ & 22.2 & 23.9 & $\mathrm{TA}^{\mathrm{e}}$ & 23.0 & 24.8 & $\mathrm{CT}^{\mathrm{e}}$ & 24.3 & 23.9 & GA \\
\hline 24 & $\mathrm{Pi}-07$ & P. infestans & US-8 & 21.0 & - & AA & 21.1 & $35.6^{\mathrm{d}}$ & $\mathrm{TT}$ & 21.5 & 31.4 & $\mathrm{CC}$ & 33.3 & 23.0 & AA \\
\hline 25 & LRC-6 & P. infestans & US-11 & 24.5 & - & AA & 24.6 & - & TT & 24.9 & $34.7^{\mathrm{d}}$ & $\mathrm{CC}$ & 24.8 & - & GG \\
\hline 26 & LRC-1 & P. infestans & US-8 & 26.1 & $32.1^{\mathrm{d}}$ & AA & 24.9 & - & $\mathrm{TT}$ & 26.1 & 33.4 & $\mathrm{CC}$ & 40.7 & 26.5 & AA \\
\hline 27 & DAOM 236393 & P. quercina & N/A & - & - & N/A & - & - & N/A & $34.4^{\mathrm{d}}$ & $36.9^{d}$ & N/A & - & - & N/A \\
\hline 28 & P3009 & P. mirabilis & N/A & - & - & N/A & $32.9^{\mathrm{d}}$ & - & N/A & 31.1 & $38.4^{\mathrm{d}}$ & $\mathrm{CC}$ & - & - & N/A \\
\hline 29 & BR 518 & P. citricola & N/A & - & - & N/A & - & - & N/A & $34.2^{\mathrm{d}}$ & $40.9^{\mathrm{d}}$ & N/A & - & - & N/A \\
\hline 30 & CBS 101553 & P. ramorum & N/A & - & - & N/A & - & $33.0^{\mathrm{d}}$ & N/A & $34.7^{\mathrm{d}}$ & $35.3^{\mathrm{d}}$ & N/A & - & - & N/A \\
\hline 31 & N/A & N/A & Negative & - & - & N/A & - & - & N/A & $34.0^{\mathrm{d}}$ & $35.8^{\mathrm{d}}$ & N/A & - & - & N/A \\
\hline
\end{tabular}

${ }^{a} \mathrm{Ct}$ set manually at 0.05 . The three genotypes at the ASO PUA-570 assay are diagnostic of $P$. infestans genotypes. Symbols: $-\mathrm{indicates} \mathrm{Ct}>45$ and N/A $=$ not applicable.

b Isolates were considered heterozygous when they displayed a difference of $\leq 4 \mathrm{Ct}$ at a given ASO primer pair, and homozygous when they displayed a difference of $\geq 7 \mathrm{Ct}$, with one exception shown in bold font. This exception displayed a difference of $\geq 6 \mathrm{Ct}$ and was considered homozygous.

${ }^{c}$ Nucleotide in parentheses is the complement of the target single-nucleotide polymorphism and is shown in the sense orientation.

${ }^{\mathrm{d}}$ Combined analyses of $\mathrm{Ct}$ values and melt curves revealed nonspecific amplifications.

e Indicates the diagnostic genotypes at each of the ASO-PCR assays. 
$5 \mathrm{ng} / \mu \mathrm{l}$ to $5 \mathrm{fg} / \mu \mathrm{l}$. Standard curves were replicated three times for each dilution for each optimized ASO primer pair.

Test of infected plant material. Infected plant material from leaf inoculation experiments performed at Agriculture and Agri-Food Canada (St-Jean-sur-Richelieu, QC, Canada) was used to test the nine optimized ASO-PCR assays developed in this study with environmental material. $P$. infestans isolates of US-23 and US-24 genotypes (PiUS23NB and Pi2011-072NB, respectively) were used to inoculate leaves of $S$. tuberosum 'Russet Burbank'. P. infestans isolates were cultured on Rye B agar medium (Caten and Jinks 1968) at room temperature with a natural photoperiod for 7 to 25 days until sporulation, prior to inoculation. Once sporulation occurred, distilled water was poured over the petri dishes, and the sporangia were suspended by gently agitating the petri dishes. The suspension was quickly transferred to a spray bottle for inoculation onto potato leaves. For each genotype, between 3 and 12 potato plants were inoculated by spraying the sporangia solution onto the two sides of each leaf. Inoculated plants were each covered with a plastic bag to maintain $100 \%$ relative humidity $(\mathrm{RH})$. Potato plants were grown in the dark at $18^{\circ} \mathrm{C}$ and $100 \% \mathrm{RH}$ for $24 \mathrm{~h}$. After this period, the plastic bags were removed, the temperature was kept at $18^{\circ} \mathrm{C}, \mathrm{RH}$ was adjusted to $95 \%$, and light was adjusted to a photoperiod of $10 \mathrm{~h}$ /day. After 7 days, potato plants were examined for infection by $P$. infestans. For four inoculated leaves/plants, approximately three discs were cut at the leading edge of the infection site with a number 4 cork borer, and kept at $-20^{\circ} \mathrm{C}$ until DNA extraction. DNA was extracted from infected plant material (4 discs/tube) using the DNeasy Plant Mini Kit (Qiagen Sciences, Germantown, MD) following the manufacturer's recommendations. DNA was then purified using the QuickPick SML Plant DNA magnetic particle purification kit (BioNobile, Turku, Finland) and diluted 1/100. For each of the nine optimized assays, PCR assays were performed at the optimal annealing temperatures listed in Table 3 with the PCR conditions described above. All reactions were run on a StepOne Real-Time PCR System and data were analyzed with StepOne Software, version 2.3.

\section{Results}

DNA polymorphisms. Among the 28 loci selected for PCR amplification and sequencing on the basis of sufficient primer and sequence information, five nuclear genes and eight flanking microsatellite regions generated a single amplification product, were variable, and did not contain indels (Table 2). These loci were mined for SNP among one or more of the dominant Canadian P. infestans genotypes. The number of SNP found at each locus and the number of SNP showing variation among one or more of the five dominant Canadian genotypes are represented in Table 2 . All of the sequences produced for these loci were deposited in GenBank (Table 2).

Optimization of ASO-PCR assays. ASO primers were designed to amplify specifically the alleles of one or more dominant Canadian $P$. infestans genotypes at 14 of the 50 variable SNP identified from

Table 5. (continued from preceding page)

\begin{tabular}{|c|c|c|c|c|c|c|c|c|c|c|c|c|c|c|c|}
\hline \multicolumn{3}{|c|}{ RevASO Ras-98 } & \multicolumn{3}{|c|}{ ASO Ras-376 } & \multicolumn{3}{|c|}{ RevASO 3332F-225 } & \multicolumn{3}{|c|}{ RevASO 3332F-258 } & \multicolumn{3}{|c|}{ RevASO 3332F-344 } & \multirow[b]{2}{*}{ Overall profile } \\
\hline $\mathbf{C}(\mathbf{G})^{\mathrm{c}}$ & $\mathbf{A}(\mathbf{T})$ & Type & $\mathbf{T}$ & $\mathbf{C}$ & Type & $\mathbf{C}(\mathbf{G})$ & $\mathbf{G}(\mathbf{C})$ & Type & $\mathbf{G}(\mathbf{C})$ & $\mathbf{T}(\mathbf{A})$ & Type & $\mathbf{G}(\mathbf{C})$ & $\mathbf{A}(\mathbf{T})$ & Type & \\
\hline 21.5 & $32.4^{\mathrm{d}}$ & GG & 20.7 & 21.5 & $\mathrm{TC}$ & 21.6 & 34.4 & GG & 20.9 & 32.7 & $\mathrm{CC}$ & 21.8 & 25.3 & $\mathrm{CT}^{\mathrm{e}}$ & US-8 \\
\hline 22.7 & 24.2 & $\mathrm{GT}^{\mathrm{e}}$ & 21.4 & 22.4 & $\mathrm{TC}$ & 22.7 & $43.5^{\mathrm{d}}$ & GG & 22.3 & 23.2 & $\mathrm{CA}^{\mathrm{e}}$ & 25.1 & 34.3 & $\mathrm{CC}$ & US-11 \\
\hline 22.5 & $32.5^{\mathrm{d}}$ & GG & 22.9 & 21.7 & $\mathrm{TC}$ & 23.2 & 26.0 & $\mathrm{GC}^{\mathrm{e}}$ & 21.9 & 36.5 & $\mathrm{CC}$ & 22.1 & 34.4 & $\mathrm{CC}$ & US-23 \\
\hline 22.9 & $30.5^{\mathrm{d}}$ & GG & 23.1 & 22.2 & $\mathrm{TC}$ & 24.1 & 26.8 & $\mathrm{GC}^{\mathrm{e}}$ & 22.5 & $40.4^{\mathrm{d}}$ & $\mathrm{CC}$ & 22.9 & 35.1 & $\mathrm{CC}$ & US-23 \\
\hline 25.0 & $28.3^{\mathrm{d}}$ & GG & 24.5 & 25.9 & $\mathrm{TC}$ & 26.5 & - & GG & 24.3 & - & $\mathrm{CC}$ & 25.8 & 29.2 & $\mathrm{CT}^{\mathrm{e}}$ & US-8 \\
\hline 24.8 & $34.5^{\mathrm{d}}$ & GG & 24.1 & 25.4 & $\mathrm{TC}$ & 25.6 & - & GG & 24.1 & - & $\mathrm{CC}$ & 25.2 & 29.3 & $\mathrm{CT}^{\mathrm{e}}$ & US-8 \\
\hline 27.3 & 27.8 & $\mathrm{GT}^{\mathrm{e}}$ & 25.9 & 32.3 & $\mathrm{TT}^{\mathrm{e}}$ & 27.7 & - & GG & 29.0 & 27.1 & $\mathrm{CA}^{\mathrm{e}}$ & 27.1 & $41.1^{\mathrm{d}}$ & $\mathrm{CC}$ & Other $P$. infestans \\
\hline 22.9 & $31.8^{\mathrm{d}}$ & GG & 22.0 & 23.2 & $\mathrm{TC}$ & 23.6 & 26.3 & $\mathrm{GC}^{\mathrm{e}}$ & 22.0 & 31.1 & $\mathrm{CC}$ & 22.7 & 35.1 & $\mathrm{CC}$ & US-24 \\
\hline 21.7 & 22.0 & $\mathrm{GT}^{\mathrm{e}}$ & 19.3 & 28.9 & $\mathrm{TT}^{\mathrm{e}}$ & 20.8 & $35.6^{\mathrm{d}}$ & GG & 20.0 & $40.3^{d}$ & $\mathrm{CC}$ & 20.7 & 33.5 & $\mathrm{CC}$ & US-22 \\
\hline 22.6 & $30.6^{\mathrm{d}}$ & GG & 22.0 & 23.2 & $\mathrm{TC}$ & 23.9 & 26.4 & $\mathrm{GC}^{\mathrm{e}}$ & 22.0 & - & $\mathrm{CC}$ & 22.7 & 36.4 & $\mathrm{CC}$ & US-24 \\
\hline 22.7 & 24.0 & $\mathrm{GT}^{\mathrm{e}}$ & 21.3 & 22.3 & $\mathrm{TC}$ & 22.1 & - & GG & 22.0 & 23.1 & $\mathrm{CA}^{\mathrm{e}}$ & 22.1 & 35.0 & $\mathrm{CC}$ & US-11 \\
\hline- & $30.4^{\mathrm{d}}$ & N/A & - & - & N/A & $26.2^{\mathrm{d}}$ & $39.0^{\mathrm{d}}$ & N/A & $40.2^{\mathrm{d}}$ & $37.1^{\mathrm{d}}$ & N/A & $36.2^{\mathrm{d}}$ & $35.9^{\mathrm{d}}$ & $\mathrm{N} / \mathrm{A}$ & Not $P$. infestans \\
\hline $39.4^{\mathrm{d}}$ & $33.6^{\mathrm{d}}$ & N/A & $28.3^{\mathrm{d}}$ & $18.2^{\mathrm{d}}$ & N/A & $17.8^{\mathrm{d}}$ & $38.8^{\mathrm{d}}$ & N/A & 35.3 & $39.9^{d}$ & $\mathrm{CC}$ & 31.4 & $38.8^{\mathrm{d}}$ & $\mathrm{CC}$ & Not $P$. infestans \\
\hline 26.9 & 28.6 & $\mathrm{GT}^{\mathrm{e}}$ & 25.8 & 26.9 & $\mathrm{TC}$ & 27.7 & - & GG & 26.0 & 27.5 & $\mathrm{CA}^{\mathrm{e}}$ & 25.7 & $41.0^{\mathrm{d}}$ & $\mathrm{CC}$ & US-11 \\
\hline 26.3 & - & GG & 24.8 & 34.4 & $\mathrm{TT}^{\mathrm{e}}$ & 25.2 & - & GG & 25.1 & 26.2 & $\mathrm{CA}^{\mathrm{e}}$ & 25.9 & $35.7^{\mathrm{d}}$ & $\mathrm{CC}$ & Other $P$. infestans \\
\hline 22.8 & 24.1 & $\mathrm{GT}^{\mathrm{e}}$ & 21.1 & 22.4 & $\mathrm{TC}$ & 22.7 & - & GG & 22.0 & 23.1 & $\mathrm{CA}^{\mathrm{e}}$ & 22.2 & 34.3 & $\mathrm{CC}$ & US-11 \\
\hline 21.5 & 22.0 & $\mathrm{GT}^{\mathrm{e}}$ & 19.2 & 29.1 & $\mathrm{TT}^{\mathrm{e}}$ & 20.6 & $36.9^{d}$ & GG & 20.0 & $36.8^{d}$ & $\mathrm{CC}$ & 20.6 & 33.1 & $\mathrm{CC}$ & US-22 \\
\hline 21.5 & $31.2^{\mathrm{d}}$ & GG & 20.6 & 21.3 & $\mathrm{TC}$ & 21.6 & - & GG & 20.8 & - & $\mathrm{CC}$ & 21.9 & 25.2 & $\mathrm{CT}^{\mathrm{e}}$ & US-8 \\
\hline 25.6 & - & GG & 25.1 & 24.9 & $\mathrm{TC}$ & 25.3 & - & GG & 24.9 & - & $\mathrm{CC}$ & 25.9 & $44.9^{\mathrm{d}}$ & $\mathrm{CC}$ & Other $P$. infestans \\
\hline- & $33.0^{\mathrm{d}}$ & N/A & - & - & N/A & $30.2^{\mathrm{d}}$ & - & $\mathrm{N} / \mathrm{A}$ & - & - & N/A & $30.9^{\mathrm{d}}$ & $34.5^{\mathrm{d}}$ & N/A & Not $P$. infestans \\
\hline- & $32.5^{\mathrm{d}}$ & N/A & $33.0^{\mathrm{d}}$ & $24.1^{\mathrm{d}}$ & N/A & $30.0^{\mathrm{d}}$ & - & N/A & - & - & N/A & - & $39.8^{\mathrm{d}}$ & N/A & Not $P$. infestans \\
\hline 24.1 & $37.1^{\mathrm{d}}$ & GG & 24.9 & 23.9 & $\mathrm{TC}$ & 26.5 & 29.1 & $\mathrm{GC}^{\mathrm{e}}$ & 24.2 & - & $\mathrm{CC}$ & 24.7 & 37.3 & $\mathrm{CC}$ & US-23 \\
\hline 22.4 & $32.1^{\mathrm{d}}$ & GG & 22.8 & 21.8 & $\mathrm{TC}$ & 23.6 & 26.5 & $\mathrm{GC}^{\mathrm{e}}$ & 21.9 & $37.3^{\mathrm{d}}$ & $\mathrm{CC}$ & 22.6 & 35.2 & $\mathrm{CC}$ & US-23 \\
\hline 21.5 & $31.5^{\mathrm{d}}$ & GG & 20.9 & 21.7 & $\mathrm{TC}$ & 21.7 & 34.2 & GG & 20.9 & $38.9^{d}$ & $\mathrm{CC}$ & 21.8 & 25.4 & $\mathrm{CT}^{\mathrm{e}}$ & US-8 \\
\hline 25.0 & 27.0 & $\mathrm{GT}^{\mathrm{e}}$ & 24.4 & 24.8 & $\mathrm{TC}$ & 24.9 & - & GG & 24.3 & 25.8 & $\mathrm{CA}^{\mathrm{e}}$ & 24.6 & 37.5 & $\mathrm{CC}$ & US-11 \\
\hline 25.7 & $30.9^{d}$ & GG & 25.3 & 25.8 & $\mathrm{TC}$ & 27.0 & - & GG & 25.2 & - & $\mathrm{CC}$ & 25.9 & 29.8 & $\mathrm{CT}^{\mathrm{e}}$ & US-8 \\
\hline- & - & N/A & - & - & N/A & $29.2^{\mathrm{d}}$ & - & $\mathrm{N} / \mathrm{A}$ & - & - & $\mathrm{N} / \mathrm{A}$ & $31.7^{\mathrm{d}}$ & $34.8^{\mathrm{d}}$ & N/A & Not $P$. infestans \\
\hline $30.9^{\mathrm{d}}$ & - & N/A & - & 29.4 & $\mathrm{CC}$ & 34.8 & - & GG & 34.2 & - & $\mathrm{CC}$ & 32.7 & - & $\mathrm{CC}$ & Not $P$. infestans \\
\hline- & $30.7^{d}$ & N/A & - & - & N/A & $28.9^{d}$ & - & N/A & - & - & N/A & $35.4^{\mathrm{d}}$ & $42.5^{\mathrm{d}}$ & N/A & Not $P$. infestans \\
\hline $32.0^{\mathrm{d}}$ & $31.2^{\mathrm{d}}$ & N/A & - & - & N/A & $38.0^{\mathrm{d}}$ & - & N/A & - & - & N/A & $39.3^{\mathrm{d}}$ & - & N/A & Not $P$. infestans \\
\hline $35.0^{\mathrm{d}}$ & $36.5^{\mathrm{d}}$ & N/A & - & - & N/A & $37.9^{d}$ & - & N/A & - & - & N/A & - & - & N/A & Negative \\
\hline
\end{tabular}


the sequencing results (Table 3; Supplementary Table S2) based on each locus position in the sequence, the requirements for designing primers, and the relative abundance of SNP specific to one or more of the dominant genotypes in Canada. For the nine optimized ASO-PCR assays listed in Table 3, P. infestans isolates presented the expected genotypes for at least one of the annealing temperatures tested. Five ASO-PCR assays targeted only one genotype: ASO PUA-120 and -225 were specific to isolates of US-23, ASO Ras-376 was specific to isolates of US-22, RevASO 3332F-258 was specific to isolates of US-11, and RevASO 3332F-344 was specific to isolates of US-8 (Table 4). The four remaining assays targeted two or more genotypes: ASO Arp23-182 targeted US-22/US-23 genotypes, RevASO Ras-98 targeted US-11/US-22 genotypes, RevASO 3332F-225 targeted US-23/US-24 genotypes, and ASO PUA-570 targeted three genotypes (US-8/US-24, US-11, and US22/US-23) (Table 4). The most significant cycle threshold (Ct) differences were obtained at the optimal temperatures reported in Table 3, with $>7 \mathrm{Ct}$ difference observed for homozygous genotypes at all loci except ASO PUA-570 (Supplementary Table S3). Melting curve analyses also showed a single peak for the nine optimized assays, indicating specific amplification at all loci (Supplementary Table S4). Therefore, for a given ASO-PCR assay, samples were considered heterozygous when the difference in $\mathrm{Ct}$ values between both primers was $\leq 4$ and homozygous when the difference in $\mathrm{Ct}$ values was $\geq 7$.

For the five other ASO-PCR assays listed in Supplementary Table $\mathrm{S} 2$, either the $P$. infestans isolates did not produce the expected genotype profile at any of the temperatures tested or the $\mathrm{Ct}$ differences were not large enough to discriminate homozygotes from heterozygotes. Three of these assays targeted only one genotype: RevASO Ras-136 and ASO Ras-260 were specific to isolates of US-11 while RevASO 3332-312 was specific to isolates of US-8. The two other assays each targeted three genotypes: ASO PUA-426 targeted US-8/US-24, US-11, and US-22/US-23 isolates and RevASO Trp1-136 targeted US-8/US-22, US-11/US-24, and US-23 isolates. Because these five assays gave unreliable results, they were not investigated further.
Validation of nine ASO-PCR assays. The nine optimized ASOPCR assays revealed unique profiles for all five dominant Canadian $P$. infestans genotypes. Expected genotypes and profiles for each of the assays are listed in Table 4. No false assignments were observed in the blind tests and all Canadian genotypes were identified correctly (Table 5). The three other $P$. infestans genotypes included in the panel (US-1, US-6, and US-7) also displayed unique profiles for the nine ASO-PCR assays. These samples were identified as other $P$. infestans genotypes in the overall profile (Table 5). For most of the assays, no specific amplifications were observed for the other Phytophthora spp. tested, thus enabling their identification as not $P$. infestans isolates. Specific amplifications were obtained in $\leq 3$ of the assays for $P$. cactorum and $P$. mirabilis, two species more closely related to $P$. infestans than the other Phytophthora spp. included in the panel (Table 5). Combined analyses of $\mathrm{Ct}$ values and melt curves revealed nonspecific amplifications for all negative samples that had positive Ct values (Table 5; Supplementary Table S5).

The nine optimized ASO-PCR assays also revealed unique profiles for 11 of the 17 recombinant $P$. infestans isolates tested (Table 6). These combinations of genotypes were not observed in the blind panel. A new genotype (TT) not observed previously was obtained with the ASO Arp23-182 locus for isolate US100034, and confirmed by sequencing (data not shown). As observed for the blind test, combined analyses of $\mathrm{Ct}$ values and melt curves revealed nonspecific amplifications for negative samples that had positive $\mathrm{Ct}$ values (Table 6; data not shown for melt curves).

Standard curves of ASO primer pairs. The limit of detection of the nine optimized ASO-PCR assays ranged from 50 to $500 \mathrm{fg} / \mu \mathrm{l}$ (Table 3) for at least one of the three replicated dilutions per assay. Efficiency ranged from 0.84 to 1.16 , with $R^{2}$ from 0.98 to 0.99 (Table 3 ).

Test of infected plant material. DNA extracted from potato leaves infected with $P$. infestans US-24 (Table 1) presented the expected genotype for all ASO markers tested (Supplementary Table S6). In addition, DNA extracted from potato leaves infected with $P$. infestans US-23 presented the expected genotype for all markers except two (ASO Arp23-182 and RevASO 3332F-225). For the latter markers, the genotypes detected were not those expected and

Table 6. Cycle threshold $(\mathrm{Ct})$ values, genotypes, and overall profile obtained for possible recombinant Phytophthora infestans isolates from the United States and Canada for nine optimized allele-specific oligonucleotide polymerase chain reaction (ASO-PCR) assays ${ }^{\mathrm{a}}$

\begin{tabular}{|c|c|c|c|c|c|c|c|c|c|c|c|c|c|c|c|c|}
\hline \multirow[b]{2}{*}{ Isolate } & \multirow[b]{2}{*}{ Genotype } & \multicolumn{3}{|c|}{ ASO Arp23-182 } & \multicolumn{3}{|c|}{ ASO PUA-120 } & \multicolumn{3}{|c|}{ ASO PUA-225 } & \multicolumn{3}{|c|}{ ASO PUA-570 } & \multicolumn{3}{|c|}{ RevASO Ras-98 } \\
\hline & & $\mathbf{A}$ & $\mathbf{T}$ & Type $^{c}$ & $\mathbf{T}$ & $\mathbf{A}$ & Type & $\mathbf{C}$ & $\mathbf{T}$ & Type & $\mathbf{G}$ & $\mathbf{A}$ & Type & $\mathbf{C}(\mathbf{G})^{\mathrm{d}}$ & $\mathbf{A}(\mathbf{T})$ & Type \\
\hline US110093 & GDT-06 & 25.3 & - & AA & 20.3 & 40.1 & TT & 22.4 & 35.3 & $\mathrm{CC}$ & 40.0 & 27.3 & AA & 23.2 & - & GG \\
\hline US110074 & GDT-04 & 26.8 & 25.0 & $\mathrm{AT}^{\mathrm{e}}$ & 20.2 & 32.8 & TT & 22.3 & 35.2 & $\mathrm{CC}$ & 40.8 & 27.1 & AA & 24.3 & 23.6 & $\mathrm{GT}^{\mathrm{e}}$ \\
\hline US110072 & GDT-07 & 26.5 & 26.2 & $\mathrm{AT}^{\mathrm{e}}$ & 20.7 & - & $\mathrm{TT}$ & 22.7 & 34.9 & $\mathrm{CC}$ & 25.4 & 29.6 & GA & 23.2 & - & GG \\
\hline US100023 & GDT-13 & 25.5 & 24.8 & $\mathrm{AT}^{\mathrm{e}}$ & 19.8 & 35.6 & TT & 22.4 & 34.0 & $\mathrm{CC}$ & 24.5 & 29.6 & GA & 22.0 & 42.8 & GG \\
\hline US110061 & GDT-05 & 24.0 & - & AA & 19.4 & 34.9 & TT & 21.5 & 34.0 & $\mathrm{CC}$ & 24.5 & 28.2 & GA & 22.6 & 23.7 & $\mathrm{GT}^{\mathrm{e}}$ \\
\hline US100033 & GDT-16 & 24.3 & - & AA & 19.7 & 35.2 & TT & 21.6 & 33.6 & $\mathrm{CC}$ & 41.0 & 26.9 & AA & 21.5 & - & GG \\
\hline US100034 & GDT-20 & - & 23.2 & TT & 18.9 & 39.1 & TT & 21.6 & 34.9 & $\mathrm{CC}$ & 24.2 & 27.3 & GA & 21.6 & - & GG \\
\hline US110086 & GDT-03 & 23.9 & - & AA & 19.3 & 38.8 & TT & 21.6 & 33.8 & $\mathrm{CC}$ & 24.1 & 27.1 & GA & 22.7 & 24.2 & $\mathrm{GT}^{\mathrm{e}}$ \\
\hline US110085 & GDT-11 & 25.7 & - & AA & 20.4 & - & TT & 22.1 & 34.4 & $\mathrm{CC}$ & 24.9 & 29.8 & GA & 23.6 & 23.7 & $\mathrm{GT}^{\mathrm{e}}$ \\
\hline US100029 & GDT-12 & 24.2 & - & AA & 19.5 & 37.6 & TT & 21.8 & 33.7 & $\mathrm{CC}$ & 24.0 & 29.3 & GA & 22.6 & 24.2 & $\mathrm{GT}^{\mathrm{e}}$ \\
\hline US100032 & GDT-15 & 24.0 & - & AA & 19.8 & 36.7 & TT & 22.2 & 34.8 & $\mathrm{CC}$ & 39.6 & 28.0 & AA & 22.7 & - & GG \\
\hline US110084 & GDT-01 & 26.9 & 26.8 & $\mathrm{AT}^{\mathrm{e}}$ & 21.5 & 37.0 & TT & 23.3 & 37.3 & $\mathrm{CC}$ & 26.4 & 30.2 & GA & 25.0 & 24.7 & $\mathrm{GT}^{\mathrm{e}}$ \\
\hline US100019 & GDT-19 & 26.1 & 24.7 & $\mathrm{AT}^{\mathrm{e}}$ & 20.4 & 37.9 & TT & 21.9 & 34.5 & $\mathrm{CC}$ & 24.5 & 28.6 & GA & 22.0 & 42.5 & GG \\
\hline US110064 & GDT-02 & 24.7 & 24.7 & AA & 20.2 & 37.1 & TT & 22.5 & 36.3 & $\mathrm{CC}$ & 24.7 & 28.8 & GA & 23.4 & 24.2 & $\mathrm{GT}^{\mathrm{e}}$ \\
\hline US110092 & GDT-17 & 24.4 & $\longrightarrow$ & AA & 19.8 & 36.9 & TT & 21.9 & 33.5 & $\mathrm{CC}$ & 39.6 & 28.1 & AA & 23.2 & 24.5 & $\mathrm{GT}^{\mathrm{e}}$ \\
\hline US110071 & GDT-08 & 26.6 & 24.5 & $\mathrm{AT}^{\mathrm{e}}$ & 19.7 & 36.5 & TT & 21.6 & 33.5 & $\mathrm{CC}$ & 25.5 & 26.8 & GA & 21.8 & - & GG \\
\hline US110082 & GDT-09 & 24.7 & - & AA & 21.3 & 37.1 & $\mathrm{TT}$ & 23.0 & 36.3 & $\mathrm{CC}$ & 43.4 & 29.0 & $\mathrm{AA}$ & 21.9 & 40.8 & GG \\
\hline Negative & N/A & - & - & N/A & - & - & N/A & - & $42.7^{\mathrm{f}}$ & N/A & - & - & N/A & - & $40.5^{\mathrm{f}}$ & N/A \\
\hline
\end{tabular}

\footnotetext{
a Symbols: - indicates $\mathrm{Ct}>45$ and N/A = not applicable.

b The three genotypes at this ASO-PCR assay are diagnostic of $P$. infestans genotypes.

${ }^{\mathrm{c}}$ Isolates were considered heterozygous when they displayed a difference of $\leq 4 \mathrm{Ct}$ at a given ASO primer pair, and homozygous when they displayed a difference of $\geq 7 \mathrm{Ct}$ with three exceptions shown in bold font. These exceptions displayed a difference of approximately $5 \mathrm{Ct}$ and were considered heterozygous.

d Nucleotide in parentheses is the complement of the target single-nucleotide polymorphism and is shown in the sense orientation.

e Indicates the diagnostic genotypes at each of the ASO-PCR assays.

${ }^{\mathrm{f}}$ Combined analyses of $\mathrm{Ct}$ values and melt curves revealed nonspecific amplifications for the negative control samples that showed positive $\mathrm{Ct}$ values.
} 
represented new mutations for this lineage, as confirmed by sequencing (data not shown). Melting curve analyses also showed a single peak, indicating specific amplification at all loci for both genotypes tested (data not shown).

\section{Discussion}

In this study, several SNP were identified in nuclear genes and flanking microsatellite regions variable among a collection of $P$. infestans genotypes. Nine of these SNP were used to develop ASO-PCR assays that enabled identification of the five most dominant genotypes present in Canada in recent years. These assays were conducted using real-time PCR assays, ensuring a high level of sensitivity without the need to start with large amounts of sample material to test. Indeed, the limit of detection of the assays ranged from 50 to $500 \mathrm{fg} / \mu \mathrm{l}$, which is in the range expected for assays located in single-copy genes or regions, as shown with $P$. ramorum (Bilodeau et al. 2007b, 2009). Also, the tests performed demonstrated that this technology can be adapted easily to different real-time PCR platforms.

From the 13 loci mined for SNP in this study, 50 sites showed variation among dominant Canadian $P$. infestans genotypes. However, SNP variation was not distributed evenly among genotypes. A greater abundance of SNP was found in US-11, US-22, and US-23 genotypes than in US-8 and US-24 genotypes. Thus, many SNP were not chosen for primer design because they could not differentiate US-8 and US-24 genotypes. Although US-8 and US-24 genotypes have distinct RFLP, allozymes, and microsatellite profiles (Danies et al. 2013; Fry et al. 2013; Hu et al. 2012; Kalischuk et al. 2012; Peters et al. 2014), a neighbor-joining tree built from microsatellite markers showed that these genotypes are very close genetically compared with US-11, US-22, and US-23 genotypes (Fry et al. 2013). Even though the nine markers developed in this study distinguish all dominant Canadian $P$. infestans genotypes, development of more markers specific to US-8 and US-24 is desirable. Additional SNP distinguishing these two genotypes could be obtained by doing a comparative analysis of their transcriptomes. Indeed, these genotypes present different phenotypic characteristics such as mating type and sensitivity to mefenoxam (Danies et al. 2013; Fry et al. 2013; $\mathrm{Hu}$ et al. 2012; Kalischuk et al. 2012; Peters et al. 2014), differences that should be reflected in their transcriptomes.

Although limited variation was found between US-8 and US-24 genotypes, plenty of SNP were available to differentiate US-11, US-22, and US-23 genotypes. Thus, there is some redundancy in the genotypes detected by the nine optimized ASO-PCR assays. For example, US-23 genotype is detected specifically by two ASO-PCR assays, ASO PUA-120 and -225. However, this genotype can also be detected with two other assays, ASO Arp23-182 and RevASO 3332F-225. This redundancy means that not all the assays are needed to identify the five dominant Canadian $P$. infestans genotypes. A good example is provided by the new mutations found in the US-23 genotype used in the inoculation experiment for two of the ASO-PCR assays, ASO Arp23-182 and RevASO 3332F-225. This genotype was still identified as US-23 despite the mutations, based on the profile exhibited with the seven other ASO-PCR assays. For routine usage, a subset of assays can be performed first, followed by confirmation of the profiles with the other markers, as needed by end users.

The ASO-PCR assays developed in this study were designed specifically to distinguish the five $P$. infestans genotypes dominant in Canada over the last 5 to 6 years (Kalischuk et al. 2012; Kawchuk et al. 2011; Peters et al. 2014). However, this marker system based on SNP variation is universal and could easily be extended to encompass new genotypes or genotypes present in other countries, as suggested by Abbott et al. (2010). For example, the nine optimized ASO-PCR assays distinguished the US-1, US-6, and US-7 genotypes included in the blind test in this study by producing unique profiles for each. The ASO-PCR assays also provided unique profiles for 11 of 17 possibly recombinant isolates found in Canada and the eastern United States. Theoretically, these markers could detect 19,683 possible unique genotype combinations (three possible genotypes for each of nine markers $=3^{9}$ ). However, the number of possible unique genotype combinations is likely to be less with these specific assays, because eight markers differentiated only two genotypes. Even with 768 possible unique genotype combinations $\left(2^{8} \times 3\right)$, this should still encompass most of the $P$. infestans genotypic variation, at least in regions where reproduction is clonal. For example, in North America, where $P$. infestans

Table 6. (continued from preceding page)

\begin{tabular}{|c|c|c|c|c|c|c|c|c|c|c|c|c|}
\hline \multicolumn{3}{|c|}{ ASO Ras-376 } & \multicolumn{3}{|c|}{ RevASO 3332F-225 } & \multicolumn{3}{|c|}{ RevASO 3332F-258 } & \multicolumn{3}{|c|}{ RevASO 3332F-344 } & \multirow[b]{2}{*}{ Overall profile } \\
\hline $\mathbf{T}$ & $\mathbf{C}$ & Type & $\mathbf{C}(\mathbf{G})$ & $\mathbf{G}(\mathbf{C})$ & Type & G (C) & $\mathbf{T}(\mathbf{A})$ & Type & $\mathbf{G}(\mathbf{C})$ & $\mathbf{A}(\mathbf{T})$ & Type & \\
\hline 22.8 & 22.6 & TC & 22.6 & - & GG & 18.8 & - & $\mathrm{CC}$ & 23.2 & 37.5 & $\mathrm{CC}$ & Recom A \\
\hline 22.4 & 23.3 & $\mathrm{TC}$ & 22.2 & 40.8 & GG & 18.5 & 42.4 & $\mathrm{CC}$ & 22.9 & 36.9 & $\mathrm{CC}$ & Recom B \\
\hline 23.2 & 22.8 & $\mathrm{TC}$ & 23.2 & 37.0 & GG & 19.2 & - & $\mathrm{CC}$ & 23.5 & 37.2 & $\mathrm{CC}$ & Recom C \\
\hline 21.3 & 35.0 & $\mathrm{TT}^{\mathrm{e}}$ & 21.9 & 44.6 & GG & 18.2 & 39.0 & $\mathrm{CC}$ & 22.6 & 36.5 & $\mathrm{CC}$ & Recom D \\
\hline 20.9 & 33.3 & $\mathrm{TT}^{\mathrm{e}}$ & 21.7 & 45.0 & GG & 18.0 & - & $\mathrm{CC}$ & 22.2 & 36.2 & $\mathrm{CC}$ & Recom E \\
\hline 20.6 & 34.4 & $\mathrm{TT}^{\mathrm{e}}$ & 21.5 & - & GG & 17.8 & - & $\mathrm{CC}$ & 21.8 & 35.7 & $\mathrm{CC}$ & Recom F \\
\hline 21.8 & 21.8 & $\mathrm{TC}$ & 21.4 & 38.3 & GG & 17.9 & - & $\mathrm{CC}$ & 21.9 & 35.8 & $\mathrm{CC}$ & Recom G \\
\hline 21.5 & 22.1 & $\mathrm{TC}$ & 21.4 & 41.3 & GG & 17.8 & 42.5 & $\mathrm{CC}$ & 22.2 & 35.8 & $\mathrm{CC}$ & Recom H \\
\hline 22.1 & 35.4 & $\mathrm{TT}^{\mathrm{e}}$ & 22.2 & 38.9 & GG & 18.2 & 42.0 & $\mathrm{CC}$ & 22.6 & 36.3 & $\mathrm{CC}$ & Recom E \\
\hline 21.1 & 36.5 & $\mathrm{TT}^{\mathrm{e}}$ & 21.7 & 42.4 & GG & 18.1 & 45.0 & $\mathrm{CC}$ & 22.3 & 35.7 & $\mathrm{CC}$ & Recom E \\
\hline 21.4 & 37.1 & $\mathrm{TT}^{\mathrm{e}}$ & 22.4 & 39.6 & GG & 18.9 & 43.4 & $\mathrm{CC}$ & 22.9 & 36.8 & $\mathrm{CC}$ & Recom F \\
\hline 22.8 & 24.2 & $\mathrm{TC}$ & 23.4 & - & GG & 20.0 & - & $\mathrm{CC}$ & 24.2 & 39.3 & $\mathrm{CC}$ & Recom I \\
\hline 22.9 & 22.5 & $\mathrm{TC}$ & 22.1 & - & GG & 18.7 & - & $\mathrm{CC}$ & 22.8 & 36.4 & $\mathrm{CC}$ & Recom J \\
\hline 21.5 & 35.7 & $\mathrm{TT}^{\mathrm{e}}$ & 22.6 & - & GG & 18.8 & - & $\mathrm{CC}$ & 22.8 & 36.3 & $\mathrm{CC}$ & Recom E \\
\hline 21.2 & 36.8 & $\mathrm{TT}^{\mathrm{e}}$ & 22.6 & - & GG & 18.3 & - & $\mathrm{CC}$ & 22.6 & 36.8 & $\mathrm{CC}$ & Recom K \\
\hline 20.7 & 34.2 & $\mathrm{TT}^{\mathrm{e}}$ & 22.4 & - & GG & 18.3 & - & $\mathrm{CC}$ & 22.6 & 36.7 & $\mathrm{CC}$ & Recom D \\
\hline 21.4 & 35.5 & $\mathrm{TT}^{\mathrm{e}}$ & 22.2 & - & GG & 18.3 & 39.9 & $\mathrm{CC}$ & 22.6 & 36.0 & $\mathrm{CC}$ & Recom F \\
\hline- & - & N/A & $35.9^{\mathrm{f}}$ & - & N/A & - & - & N/A & - & - & N/A & N/A \\
\hline
\end{tabular}


reproduces clonally for the most part (Fry 2008), about 44 distinct genotypes (US-1 to US-24 and GDT-01 to GDT-20) have been reported from the United States over the last 20 years (Danies et al. 2013, 2014; Forbes et al. 1998; Goodwin et al. 1994, 1995, 1998; Hu et al. 2012; Peters et al. 2014). This marker system could also be implemented in regions where recombination is frequent, such as in Scandinavia (Brurberg et al. 2011; Sjöholm et al. 2013; Yuen and Andersson 2013). However, in these regions, more loci might be needed to increase the number of unique genotype combinations detected, which might pose a challenge for development and might not be ideal because of the increased number of assays that would be required.

The genotyping of 17 potentially recombinant $P$. infestans isolates from the eastern United States and Canada with the ASO-PCR assays revealed 11 unique profiles not observed previously, either for the five dominant $P$. infestans genotypes in Canada or for US-1, US-6, or US-7 genotypes. The variation observed for these profiles occurred mainly around the ASO-PCR assays specific to US-22 genotype, whereas the assays specific to other genotypes did not show variation. The high number of new genotype combinations observed for these markers is likely the result of segregation and suggests that US-22 might be a parental genotype for some of these isolates. This is consistent with the results of parentage exclusion analysis performed by Danies et al. (2014).

The development of ASO-PCR assays with SYBR Green technology was chosen over the design of assays with specific probes (e.g., TaqMan, minor groove binding, or lock nucleic acids) for two reasons. First, ASO-PCR assays are easier to design than hybridization probes, and second, ASO-PCR assays are of relative low cost compared with probes because they are based on SYBR Green technology. These features make development and implementation easier for end users than probe-based assays. Probe-based assays do have advantages over ASO-PCR assays in that they are usually more specific than SYBR Green assays, emitting fluorescence only when bound to a specific region of interest, not any double-stranded DNA. However, if a melting curve is included in ASO-PCR assays with SYBR Green, this can be used to check that the product amplified is specific, as demonstrated in this study. Probe-based assays do have the advantage of options for multiplexing compared with single-plex ASO-PCR assays, which was not investigated in the present study. It might be worth developing a multiplex approach to ASO-PCR assays to decrease the number of required runs and, therefore, the time required for identification of $P$. infestans genotypes. This can be crucial during late blight outbreaks. Moreover, the assays developed in this study could be modified to one or more isothermal amplification techniques. For example, the isothermal amplification technique using recombinase polymerase amplification allows multiplexing and can be used directly in the field with portable instruments, as shown by Miles et al. (2015) for the detection of Phytophthora spp.

Currently, potato growers are only informed about the genotypes of $P$. infestans present in their fields late in the season. This delay is mostly caused by the lack of cost-effective methods for genotype determination. An interesting characteristic of the ASO-PCR assays developed in this study is that they can be used on environmental samples because they only require limited amounts of starting material (Bilodeau et al. 2007a, 2009). This contrasts with RFLP methods that need large amounts of DNA as starting material (Cooke and Lees 2004) that must be harvested from pure cultures of the pathogen, which can take several weeks to months to produce (L. Kawchuk, personal communication). These markers represent a complement to existing genotyping methods for $P$. infestans, offering new perspectives such as rapid determination of genotypes from incoming inoculum monitored with spore samplers (Fall et al. 2014) or from first lesions observed in potato fields or on trap plants. Furthermore, Fall et al. (2015) showed that the infection efficiency of $P$. infestans sporangia varies among genotypes. Consequently, informed late blight management decisions should consider weather conditions, the amount of inoculum present, resistance of the incoming inoculum to fungicides, and virulence of the isolates. Tests performed on DNA extracted from infected plant material revealed that the nine ASO-PCR assays developed in this study allowed the detection and identification of the
US-23 and US-24 genotypes on potato without interference from potato leaf material. The nine markers developed will contribute to the genomic toolbox available to assess the genetic diversity of $P$. infestans at the intraspecific level. From a disease management standpoint, the markers and assays will help to select the most appropriate fungicides to use and their application frequency to manage late blight.

\section{Acknowledgments}

We thank M. Newton, D. Shearlaw, R. Vandenbeek (Pathogen Identification Research Lab, CFIA, Ottawa, Canada), A. Levasseur (AAFC, St-Jean-surRichelieu, Canada), and W. Wijekoon (AAFC, Lethbridge, Canada) for technical help; S. C. Brière (Plant Pathology Lab, CFIA, Ottawa, Canada) and M. Fal (AAFC, St-Jean-sur-Richelieu, Canada) for providing material and sharing information; and T. Rintoul (AAFC, Ottawa, Canada) for editing the manuscript. Financial support was provided by the Canadian Safety and Security Program (project number CRTI 09-462RD)

\section{Literature Cited}

Abbott, C., Gilmore, S., Lewis, C., Chapados, J., Peters, R., Platt, H., Coffey, M., and Lévesque, C. 2010. Development of a SNP genetic marker system based on variation in microsatellite flanking regions of Phytophthora infestans. Can. J. Plant Pathol. 32:440-457.

Bilodeau, G. J., Lévesque, C. A., De Cock, A. W. A. M., Brière, S. C., and Hamelin, R. C. 2007a. Differentiation of European and North American genotypes of Phytophthora ramorum by real-time polymerase chain reaction primer extension. Can. J. Plant Pathol. 29:408-420.

Bilodeau, G. J., Lévesque, C. A., De Cock, A. W. A. M., Duchaine, C., Brière, S., Uribe, P., Martin, F. N., and Hamelin, R. C. 2007b. Molecular detection of Phytophthora ramorum by real-time polymerase chain reaction using TaqMan, SYBR Green, and molecular beacons. Phytopathology 97:632-642.

Bilodeau, G. J., Martin, F. N., Coffey, M. D., and Blomquist, C. L. 2014 Development of a multiplex assay for genus-and species-specific detection of Phytophthora based on differences in mitochondrial gene order. Phytopathology 104:733-748.

Bilodeau, G. J., Pelletier, G., Pelletier, F., Hamelin, R. C., and Lévesque, C. A. 2009. Multiplex real-time polymerase chain reaction (PCR) for detection of Phytophthora ramorum, the causal agent of sudden oak death. Can. J. Plant Pathol. 31:195-210.

Blair, J. E., Coffey, M. D., and Martin, F. N. 2012. Species tree estimation for the late blight pathogen, Phytophthora infestans, and close relatives. PLoS One 7:e37003.

Bottema, C. D., and Sommer, S. S. 1993. PCR amplification of specific alleles: Rapid detection of known mutations and polymorphisms. Mutat. Res. Fundam. Mol. Mech. Mutagen. 288:93-102.

Bourke, P. M. A. 1964. Emergence of potato blight, 1843-46. Nature 203:805-808

Brumfield, R. T., Beerli, P., Nickerson, D. A., and Edwards, S. V. 2003. The utility of single nucleotide polymorphisms in inferences of population history. Trends Ecol. Evol. 18:249-256.

Brurberg, M. B., Elameen, A., Le, V. H., Nærstad, R., Hermansen, A., Lehtinen, A., Hannukkala, A., Nielsen, B., Hansen, J., and Andersson, B. 2011. Genetic analysis of Phytophthora infestans populations in the Nordic European countries reveals high genetic variability. Fungal Biol. 115:335-342.

Carter, D., Archer, S., Buck, K., Shaw, D., and Shattock, R. 1990. Restriction fragment length polymorphisms of mitochondrial DNA of Phytophthora infestans. Mycol. Res. 94:1123-1128.

Caten, C. E., and Jinks, J. L. 1968. Spontaneous variability of single isolates of Phytophthora infestans. I. Cultural variation. Can. J. Bot. 46:329-348.

Cooke, D., and Lees, A. 2004. Markers, old and new, for examining Phytophthora infestans diversity. Plant Pathol. 53:692-704.

Danies, G., Myers, K., Mideros, M. F., Restrepo, S., Martin, F. N., Cooke, D. E. L., Smart, C. D., Ristaino, J. B., Seaman, A. J., Gugino, B. K., Grünwald, N. J., and Fry, W. E. 2014. An ephemeral sexual population of Phytophthora infestans in the northeastern United States and Canada. PLoS One 9:e116354.

Danies, G., Small, I. M., Myers, K., Childers, R., and Fry, W. E. 2013. Phenotypic characterization of recent clonal lineages of Phytophthora infestans in the United States. Plant Dis. 97:873-881.

Dowley, L., and O'Sullivan, E. 1981. Metalaxyl-resistant strains of Phytophthora infestans (Mont.) de Bary in Ireland. Potato Res. 24:417-421.

Fall, M. L., Tremblay, D. M., Gobeil-Richard, M., Couillard, J., Rocheleau, H., Van der Heyden, H., Lévesque, C. A., Beaulieu, C., and Carisse, O. 2015. Infection efficiency of four Phytophthora infestans clonal lineages and DNAbased quantification of sporangia. PLoS One 10:e136312.

Fall, M. L., Van der Heyden, H., Brodeur, L., Leclerc, Y., Moreau, G., and Carisse, O. 2014. Spatiotemporal variation in airborne sporangia of Phytophthora infestans: Characterization and initiative toward improving potato late blight risk estimation. Plant Pathol. 64:178-190.

Forbes, G. A., Goodwin, S. B., Drenth, A., Oyarzun, P., Ordoñez, M. E., and Fry, W. E 1998. A global marker database for Phytophthora infestans. Plant Dis. 82:811-818.

Fry, W. 2008. Phytophthora infestans: The plant (and R gene) destroyer. Mol. Plant Pathol. 9:385-402.

Fry, W. E., Grünwald, N. J., Cooke, D., McLeod, A., Forbes, G. A., and Cao, K. 2009 Population genetics and population diversity of Phytophthora infestans. Pages 
139-164 in: Oomycete Genetics and Genomics: Diversity, Interactions, and Research Tools. K. Lamour and S. Kamoun, eds. Wiley-Blackwell, Hoboken, NJ.

Fry, W. E., McGrath, M. T., Seaman, A., Zitter, T. A., McLeod, A., Danies, G., Small, I. M., Myers, K., Everts, K., Gevens, A. J., Gugino, B. K., Johnson, S. B., Judelson, H., Ristaino, J., Roberts, P., Secor, G., Seebold, K., Jr., Snover-Clift, K., Wyenandt, A., Grünwald, N. J., and Smart, C. D. 2013. The 2009 late blight pandemic in the eastern United States - Causes and results. Plant Dis. 97:296-306.

Gagnon, M.-C., Bergeron, M.-J., Hamelin, R. C., Grünwald, N. J., and Bilodeau, G. J. 2014. Real-time PCR assay to distinguish Phytophthora ramorum lineages using the cellulose binding elicitor lectin (CBEL) locus. Can. J. Plant Pathol. 36: 367-376.

Gavino, P. D., and Fry, W. E. 2002. Diversity in and evidence for selection on the mitochondrial genome of Phytophthora infestans. Mycologia 94:781-793.

Gómez-Alpizar, L., Carbone, I., and Ristaino, J. B. 2007. An Andean origin of Phytophthora infestans inferred from mitochondrial and nuclear gene genealogies. Proc. Natl. Acad. Sci. USA 104:3306-3311.

Goodwin, S. B., Cohen, B. A., Deahl, K. L., and Fry, W. E. 1994. Migration from northern Mexico as the probable cause of recent genetic changes in populations of Phytophthora infestans in the United States and Canada. Phytopathology 84: 553-558.

Goodwin, S. B., Drenth, A., and Fry, W. E. 1992. Cloning and genetic analyses of two highly polymorphic, moderately repetitive nuclear DNAs from Phytophthora infestans. Curr. Genet. 22:107-115.

Goodwin, S. B., Smart, C. D., Sandrock, R. W., Deahl, K. L., Punja, Z. K., and Fry, W. E. 1998. Genetic change within populations of Phytophthora infestans in the United States and Canada during 1994 to 1996: Role of migration and recombination. Phytopathology 88:939-949.

Goodwin, S. B., Sujkowski, L. S., Dyer, A. T., Fry, B. A., and Fry, W. E. 1995. Direct detection of gene flow and probable sexual reproduction of Phytophthora infestans in northern North America. Phytopathology 85:473-479.

Goodwin, S. B., Sujkowski, L. S., and Fry, W. E. 1996. Widespread distribution and probable origin of resistance to metalaxyl in clonal genotypes of Phytophthora infestans in the United States and Western Canada. Phytopathology 86:793-800.

Green, E., Bain, S., Day, P., Barnett, A., Charleson, F., Jones, A., and Walker, M. 1991. Detection of human apolipoprotein E3, E2, and E4 genotypes by an allelespecific oligonucleotide-primed polymerase chain reaction assay: development and validation. Clin. Chem. 37:1263-1268.

Griffith, G. W., and Shaw, D. S. 1998. Polymorphisms in Phytophthora infestans: Four mitochondrial haplotypes are detected after PCR amplification of DNA from pure cultures or from host lesions. Appl. Environ. Microbiol. 64:4007-4014.

Grünwald, N. J., and Flier, W. G. 2005. The biology of Phytophthora infestans at its center of origin. Annu. Rev. Phytopathol. 43:171-190.

Guichoux, E., Lagache, L., Wagner, S., Chaumeil, P., Léger, P., Lepais, O., Lepoittevin, C., Malausa, T., Revardel, E., and Salin, F. 2011. Current trends in microsatellite genotyping. Mol. Ecol. Resour. 11:591-611.

Hall, T. A. 1999. BioEdit: A user-friendly biological sequence alignment editor and analysis program for Windows 95/98/NT. Nucleic Acids Symp. Ser. 41:95-98.

Haverkort, A. J., Boonekamp, P. M., Hutten, R., Jacobsen, E., Lotz, L. A. P., Kessel, G. J. T., Visser, R. G. F., and van der Vossen, E. A. G. 2008. Societal costs of late blight in potato and prospects of durable resistance through cisgenic modification. Potato Res. 51:47-57.

Hu, C.-H., Perez, F. G., Donahoo, R., McLeod, A., Myers, K., Ivors, K., Secor, G., Roberts, P. D., Deahl, K. L., and Fry, W. E. 2012. Recent genotypes of Phytophthora infestans in the eastern United States reveal clonal populations and reappearance of mefenoxam sensitivity. Plant Dis. 96:1323-1330.
Kalischuk, M. L., Al-Mughrabi, K. I., Peters, R. D., Howard, R. J., Platt, H. W., and Kawchuk, L. M. 2012. Genetic composition of Phytophthora infestans in Canada reveals migration and increased diversity. Plant Dis. 96:1729. 1735.

Kato, M., Mizubuti, E. S., Goodwin, S. B., and Fry, W. E. 1997. Sensitivity to protectant fungicides and pathogenic fitness of clonal lineages of Phytophthora infestans in the United States. Phytopathology 87:973-978.

Kawchuk, L. M., Howard, R. J., Peters, R. D., and Al-Mughrabi, K. I. 2011. First report of Phytophthora infestans genotype US23 causing late blight in Canada. Plant Dis. 95:873.

Knapova, G., and Gisi, U. 2002. Phenotypic and genotypic structure of Phytophthora infestans populations on potato and tomato in France and Switzerland. Plant Pathol. 51:641-653

Lees, A., Wattier, R., Shaw, D., Sullivan, L., Williams, N., and Cooke, D. 2006. Novel microsatellite markers for the analysis of Phytophthora infestans populations. Plant Pathol. 55:311-319.

Li, Y., Cooke, D. E., Jacobsen, E., and van der Lee, T. 2013. Efficient multiplex simple sequence repeat genotyping of the oomycete plant pathogen Phytophthora infestans. J. Microbiol. Methods 92:316-322.

Li, Y., Govers, F., Mendes, O., Testa, A., Jacobsen, E., Huang, S. W., and van der Lee, T. 2010. A new set of highly informative SSR markers for Phytophthora infestans population analysis assembled into an efficient multiplex. Mol. Ecol. Resour. 10:1098-1105.

Martin, F. N., Coffey, M. D., Zeller, K., Hamelin, R. C., Tooley, P., Garbelotto, M., Hughes, K. J. D., Kubisiak, T., Bilodeau, G. J., Levy, L., Blomquist, C. and Berger, P. H. 2009. Evaluation of molecular markers for Phytophthora ramorum detection and identification: Testing for specificity using a standardized library of isolates. Phytopathology 99:390-403.

Martin, F. N., Gloria Abad, Z., Balci, Y., and Ivors, K. 2012a. Identification and detection of Phytophthora: Reviewing our progress, identifying our needs. Plant Dis. 96:1080-1103.

Martin, F. N., Zhang, Y., Grünwald, N. J., Cooke, D. E. L., and Coffey, M. D. 2012b. Expanded analysis of $P$. infestans mitochondrial haplotypes and correlation with nuclear genotype. (Abstr.) Phytopathology 102:S4:76.

Miles, T. D., Martin, F. N., and Coffey, M. D. 2015. Development of rapid isothermal amplification assays for detection of Phytophthora spp. in plant tissue. Phytopathology 105:265-278.

Morin, P. A., Luikart, G., and Wayne, R. K. 2004. SNP in ecology, evolution and conservation. Trends Ecol. Evol. 19:208-216.

Newton, C., Graham, A., Heptinstall, L., Powell, S., Summers, C., Kalsheker, N., Smith, J., and Markham, A. 1989. Analysis of any point mutation in DNA. The amplification refractory mutation system (ARMS). Nucleic Acids Res. 17:2503-2516.

Nowicki, M., Foolad, M. R., Nowakowska, M., and Kozik, E. U. 2012. Potato and tomato late blight caused by Phytophthora infestans: An overview of pathology and resistance breeding. Plant Dis. 96:4-17.

Peters, R. D., Al-Mughrabi, K. I., Kalischuk, M. L., Dobinson, K. F., Conn, K. L., Alkher, H., Islam, M. R., Daayf, F., Lynn, J., Bizimungu, B., De Koeyer, D., Lévesque, C. A., and Kawchuk, L. M. 2014. Characterization of Phytophthora infestans population diversity in Canada reveals increased migration and genotype recombination. Can. J. Plant Pathol. 36:73-82.

Sjöholm, L., Andersson, B., Högberg, N., Widmark, A.-K., and Yuen, J. 2013 Genotypic diversity and migration patterns of Phytophthora infestans in the Nordic countries. Fungal Biol. 117:722-730.

Yuen, J., and Andersson, B. 2013. What is the evidence for sexual reproduction of Phytophthora infestans in Europe? Plant Pathol. 62:485-491. 